\title{
Selective cellular probes for mammalian thioredoxin reductase TrxR1: rational design of RX1, a modular 1,2-thiaselenane redox probe
}

\author{
Lukas Zeisel ${ }^{1}$, Jan G. Felber ${ }^{1}$, Karoline C. Scholzen ${ }^{2}$, Lena Poczka ${ }^{1}$, Dorian Cheff ${ }^{3}$, Martin S. Maier ${ }^{1}$, Qing \\ Cheng $^{2}$, Min Shen ${ }^{3}$, Matthew D. Hall ${ }^{3}$, Elias S. J. Arnér ${ }^{2,4}$, Julia Thorn-Seshold ${ }^{1}$, Oliver Thorn-Seshold ${ }^{1, *}$ \\ ${ }^{1}$ Department of Pharmacy, Ludwig-Maximilians University of Munich, Butenandtstr. 5-13, 81377 Munich, DE. \\ ${ }^{2}$ Division of Biochemistry, Department of Medical Biochemistry and Biophysics, Karolinska Institutet, Solnavägen 9, 17177 Stockholm, SE. \\ ${ }^{3}$ National Center for Advancing Translational Sciences, National Institutes of Health, 9800 Medical Center Drive, Rockville, \\ Maryland 20850, USA. \\ ${ }^{4}$ Department of Selenoprotein Research, National Institute of Oncology, 1122 Budapest, HU.
}

Cellular redox networks power a multitude of cellular processes, and are often dysregulated in pathologies including cancer and inflammatory diseases. Quantifying the turnover of the key players in redox homeostasis is crucial for understanding their physiological dynamics and for targeting them in pathologies. However, suitably selective probes for assessing specific redox enzyme activities in cells are lacking. We rationally developed the first chemical probes targeting the mammalian selenoprotein thioredoxin reductase (TrxR) while fully resisting other cellular thiols and oxidoreductases. We used a cyclic selenenylsulfide as a thermodynamically stable and kinetically reversible trigger, oriented to harness the chemistry of TrxR's unique selenolthiol active site, and integrated it into modular probes releasing arbitrary cargos upon reduction. The probes showed remarkable selenocysteine-dependent sensitivity to cytosolic TrxR1, against a panel of oxidoreductases. Lead probe RX1 also had excellent TrxR1-selective performance in cells, as cross-validated by TrxR1 knockout, selenium starvation, TrxR1 knock-in, and TrxRselective chemical inhibitors. Its background-free fluorogenicity enabled us to perform the first quantitative high-throughput live cell screen for TrxR1 inhibitors. This indicated that tempered $S_{N} A r$ electrophiles may be a more favorable drug class than classically-used electrophiles. The RX1 design is thus a robust, cellularly-validated, high-performance modular system for mammalian TrxR1. This sets the stage for in vivo imaging TrxR1 activity in health and disease, and can also drive and reorient TrxR1-inhibitor drug design. The thermodynamic and kinetic considerations behind RX1's selectivity also outline paths towards rationally-designed probes for other key players in redox biology.

The thioredoxin reductase (TrxR)-thioredoxin (Trx) system and the glutathione reductase (GR)-glutathione (GSH)-glutaredoxin (Grx) system are the two highly evolutionarily conserved "central nodes" of redox biology, which are of fundamental importance across all eukaryotes (Fig. 1a). ${ }^{1,2}$ They drive and buffer a range of biological redox reactions that are crucial to metabolism, protein folding, signaling, protein regulation, and many aspects of cellular homeostasis and stress responses. ${ }^{3,4}$ These systems are driven by reducing equivalents harvested from NADPH through the enzymes TrxR and GR, then distributed by downstream effector proteins, mainly isoenzymes of Trx and Grx, into various manifolds of dithiol/disulfidetype reactions. For all these redox enzymes, both chemocompatibility and protein-substrate binding determine their substrate scopes; these combine with subcellular compartmentalisation of the isoforms and substrates in each cascade to allow sophisticated regulation and spatial organisation of redox reactions in cells. ${ }^{5}$

Due to the fundamental importance of redox networks across biology, developing techniques to monitor and to respond to their dynamics is critical for understanding cellular physiology. Biological approaches to monitor redox biochemistry include redox-responsive ratiometric fluorescent protein fusions. These are well-established for imaging redox poise (the balance between reduced and oxidised fusion protein), and include sensors for Grxs and Trxs. ${ }^{6-8}$ However, they do not reveal the turnovers these species undergo, which are integral to a network understanding of redox homeostasis.
Additionally, since TrxR and GR are NADPH-driven enzymes, ratiometric monitoring of their redox poise is not informative about their biology, which depends on turnover. Measuring mRNA or protein expression levels is also insufficient to understand cellular redox systems, since reaction rates through networks are dynamically controlled on many levels e.g. by protein binding partners, post-translational modifications, subcellular localisation, and throttling flow from upstream reductants (or to downstream electron acceptors). Molecular probes that selectively report on the activity of individual redox-active proteins within the TrxR/Trx and GR/GSH/Grx networks would be ideal tools for studying redox biology: but no such probes exist.

Here, we sought to develop TrxR-selective molecular probes that noninvasively report TrxR activity in live cells. We focused on chemical designs to monitor enzymatic turnover by irreversible accumulation of signal, in the form of an activated probe. We additionally required the probes to be modular: i.e. the same approach should be applicable to activating arbitrary cargos, including drugs. This is of particular interest since redox dysregulation, including upregulation of TrxR activity and expression, is correlated to disease progression, severity, and resistance to conventional therapeutics, in a range of pathologies. ${ }^{9}$ These include nearly all solid tumors (hypoxia-induced gene expression shifts), as well as auto-immune and inflammatory conditions (redox signaling and microenvironment effects)..$^{10}$ Developing modular strategies to activate imaging agents (for diagnostics) as well as drugs (for redox-targeted therapeutics) could therefore be a 
powerful approach not just to understand, but also to usefully respond to cellular redox activity in health and in disease.

The challenge for small-molecule probes of dithiol/disulfide-type oxidoreductases is to distinguish selectively between proteins that perform similar chemistries. The TrxR and GR systems that are the major mammalian dithiol/disulfide reductants (Fig. 1a) have been well reviewed. ${ }^{5}$ In brief: (i) TrxRs and GRs are low-expression enzymes (ca. $20 \mathrm{nM}$ cellular concentration) that harvest electrons from NADPH to dithiol/disulfide active sites (VNVG드 motif). ${ }^{11}$ GR has high specificity for reducing glutathione disulfide (GSSG) and little activity on other species. The major TrxRs in mammals are cytosolic TrxR1 and mitochondrial TrxR2. They have a broader substrate scope than $\mathrm{GR}$, due to their additional C-terminal selenolthiol/selenenylsulfide active site ( $\underline{\mathrm{CU}}$ motif) located on an exposed flexible tail, that relays electrons from the NADPH-driven dithiol site to substrates. TrxR's native substrates include disulfide Trxs and TRP14; diverse small molecules can also be reduced (Fig. 1b). ${ }^{12,13}$ The rare selenolthiol of TrxR endows it with distinctive redox properties compared to dithiol proteins, including enhanced reaction kinetics, lowered reduction potential, and a resistance to permanently function-suppressing oxidation. ${ }^{14,15}$ (ii) Trxs and dithiol Grxs are proteins with exposed dithiol/disulfide redoxactive sites ( $\underline{\mathrm{C} x x} \underline{\mathrm{C}}$ motif) that reduce a broad scope of substrates including disulfides; they have moderate expression levels (up to ca. $10 \mu \mathrm{M})$. Trxs have lower reduction potentials and are reduced by TrxR; Grxs have higher reduction potentials and use monothiol GSH (ca. 2-5 mM in the cytosol) for recovery via a net trimolecular reaction. ${ }^{16}$

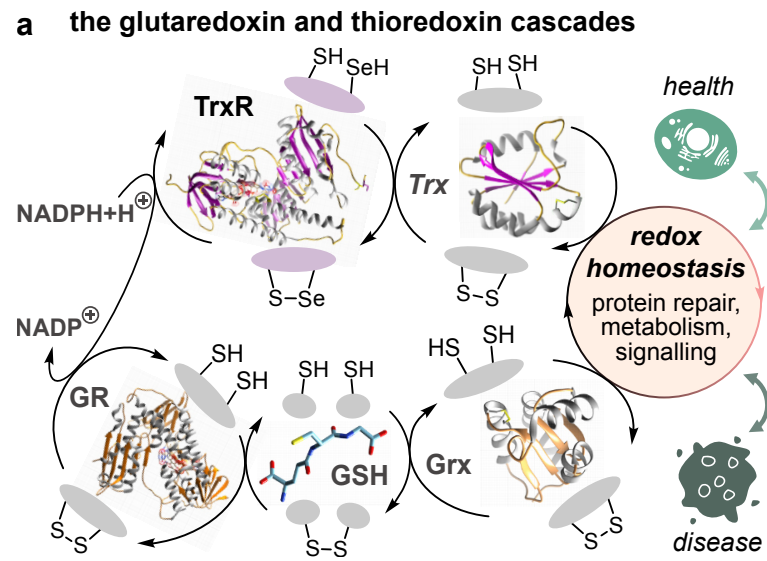

b TrxR-catalysed reduction of dichalcogenides

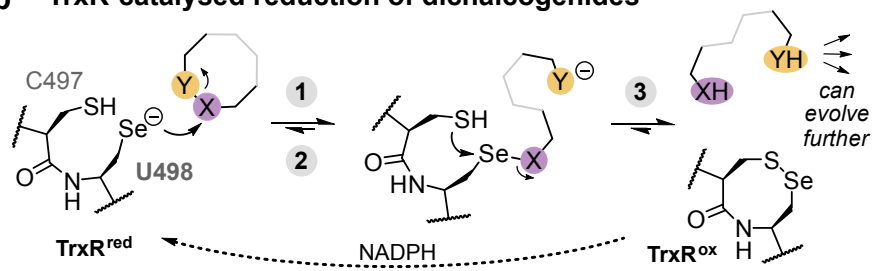

c cyclic vs. linear topology of dichalcogenide probes

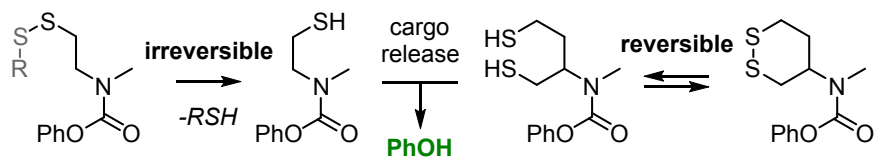

Fig. 1 Mechanistic considerations towards designing TrxR-selective probes. a, Simplified view of the central dithiol/disulfide-type redox cascades of cell biology. $\mathbf{b}$, Reaction mechanism for TrxR reducing a generalised dichalcogenide XY, using its C-terminal Sec-Cys active site. Initial exchange (1) gives an intermediate that can either evolve backwards by reforming the dichalcogenide (2) or else evolve forwards by full reduction (3) to the dichalcogenol. Recovery of TrxR by NADPH makes this a non-equilibrium process. c, Topology considerations: linear topology dichalcogenides are irreversibly committed to cargo release after monothiol exchange, whereas the exchange and reduction steps for cyclic topology dichalcogenides are reversible (full mechanism in Fig. S3).

Chemocompatible disulfide-based probes have long been explored as reporters for dithiol-type enzymes. ${ }^{17}$ Disulfide triggercargo constructs are a conceptually simple, modular turn-on design for probes (Fig. 1c). Ideally, the trigger is attached to the cargo to mask a key structural element, such that (i) the intact probe is fully deactivated (e.g. optically silent), but (ii) trigger reduction causes a cascade that irreversibly restores activity by unmasking (e.g. fluorogenesis), often by simply liberating the cargo (Fig. S1c). Analysing disulfide trigger-cargo probes' performance shows that both disulfide topology (linear or cyclic), as well as geometry (strained or stabilised), are key to cellular performance since they determine a probe's sensitivity to monothiols. ${ }^{17}$ Monothiols are highly concentrated in cells, with 1-8 mM GSH, and an even larger pool of protein thiols (PRSH). ${ }^{18,19}$ To report selectively on redox-active dithiol/disulfide proteins, probes must resist attack by this monothiol background. However, any thiol/disulfide exchange reaction upon linear-topology disulfides irreversibly commits a probe to cargo release (Fig. 1c, Fig. S3a). This makes linear topology probes nonspecifically labile to cellular monothiols, ${ }^{20-22}$ preventing them from being enzyme-selective reporters in the cellular context. ${ }^{23}$ Although some reports have used linear disulfide probes (Fig. S1a), we believed that selective reporters would require a different design. ${ }^{17}$

Cyclic-topology disulfide probes can resist triggering by monothiols in two ways. They can reform the disulfide after the initial thiol- disulfide exchange by expelling the attacking monothiol, or after reduction to the dithiol they can be re-oxidised by other disulfides in their environment: both of which prevent them from committing to cargo release (Fig. S3b; full discussion in ref. ${ }^{23}$ ). The geometry of the cyclic disulfide is critical for these reactions, and so determines the cellular performance of such probes. Strained cyclic disulfides were characterised by Whitesides in the 1990s. ${ }^{24-26}$ Noteworthily, they are kinetically labile, being rapidly and nonspecifically opened by monothiols, and opening is irreversible since disulfide strain disfavours reclosure. Thus in the cellular context, cyclic 7-membered, ${ }^{17}$ ETP-type-6-membered, ${ }^{27}$ and cyclic 5-membered disulfides ${ }^{23,28}$ cannot be selective substrates for dithiol proteins. Although probes based on cyclic 5-membered disulfides have been published and commercialised as TrxR-selective (Fig. S1a), ${ }^{29-31}$ their unselectivity and kinetic problems have been demonstrated (Fig. S1b,c) ${ }^{23,28,32-35}$ Alicyclic 6-membered disulfides (1,2-dithianes), however, are unstrained and are stable against monothiols. Monocyclic and annelated bicyclic 1,2-dithianes were recently used as reductive triggers that resist $\mathrm{GSH}^{22}$ and $\mathrm{TrxR}^{36}$, yet can be harnessed in probes that are selective for Trx (Fig. S1c) (see Supporting Note 1). ${ }^{17}$

Here, we wished to leverage these design principles to develop modular probes that resist the cellular monothiol background but would report selectively on TrxR1 activity in live cells. Analysing thermodynamics, kinetics, and reaction pathways led us to design 
novel cyclic 6-membered selenenylsulfides as reduction triggers. We developed scalable syntheses for a palette of cumulative-release fluorogenic probes with a modular design. A range of cell-free and cellular assays including genetic knockout, knock-in, chemical inhibition, selenium supplementation and depletion studies, confirmed their outstanding selectivity for reporting on cellular TrxR1. The solubilised probe $\mathbf{R X 1}$, for Trx $\underline{R} 1$-redox-probe $\underline{1}$, is a particularly rapid and stable cellular fluorescence imaging reporter of TrxR1 activity. We could apply RX1 to enable the first high-throughput selective cellular screening for TrxR inhibitors, which indicated $S_{N} A r$-based inhibitors may be favoured chemotypes for future development. RX1 offers a flexible platform for TrxR1 imaging as well as further development of TrxR probes and prodrugs; and the analysis guiding its development can light a path for other key redox-active enzymes.

\section{RESULTS}

Design of a TrxR-selective dichalcogenide trigger. We wished to design non-disulfide dichalcogenide triggers which would react selectively with TrxR's unique selenolthiol motif and give rapid cargo release even with nM TrxR, without releasing cargo with other cellular reductants - whether $\mu \mathrm{M}$ vicinal dithiols or $\mathrm{mM}$ monothiols.

Since we were designing irreversible-release probes, we first considered kinetic aspects on the path to cargo release, as relevant to selectivity. We consider that the same topology restrictions apply as in the disulfide series: ${ }^{17}$ i.e. that linear dichalcogenides are likely to be nonselective due to irreversibility of triggering under monothiol challenge. We note that two linear (non-disulfide) dichalcogenide-trigger probes have been published as selective (Fig. S1a); ${ }^{37,38}$ but according to our analysis, selectivity in the cellular context was not shown (see Supporting Note 2). We instead proceeded with cyclic-topology designs. We also considered that the same geometric factors would apply as for disulfides, ruling out strained dichalcogenides from being cellularly selective. In support of this view, Matile has shown the lability of cyclic 5-membered diselenide probes ${ }^{34}$ to nonspecific thiol exchange, mirroring that of the corresponding disulfides (Fig. S1b).

We therefore expected that only cyclic 6-membered dichalcogenides might avoid kinetically irreversible, nonspecific triggering in cells, by ensuring that monothiol attack has the potential to be reversible (discussion in ${ }^{17}$ ). This is only a partial solution however. Ensuring that highly reducing dithiol proteins e.g. Trx and Grx $(10 \mu \mathrm{M})$ or GSH (up to $8 \mathrm{mM}$ ) do not catalyse cargo release, while only ca. $20 \mathrm{nM}$ TrxR can do so, cannot be feasible by considering thermodynamics alone (e.g. Trx-reducible 1,2-dithianes resist TrxR, see Supporting Note 3). Ensuring this requires specificity either in binding or in the pathway to cargo release; and since TrxR's CU active site is on an unstructured tail, we focused instead on pathways.

Analysing reductive release pathways identified four key design aspects, which convinced us that probes based on a desymmetrised cyclic selenenylsulfide trigger $\mathbf{A}$ represent a unique TrxR-selective solution (Fig. 2a; see also Fig. S4 and Supporting Note 3). It also suggested that the thermodynamically identical regioisomers $\mathbf{G}$ (Fig. 2b) ought not be TrxR-selective, so giving highly stringent controls for our postulated specificity mechanism:

(1): Selenenylsulfides should be rapidly attacked at Se by $\operatorname{TrxR}$ selenolate: (i) Rates of selenolate attack upon the Se of a selenenylsulfide are orders of magnitude faster than those of thiolates $\left(k_{\mathrm{R} 1} \mathrm{vs} \mathrm{k}_{\mathrm{T} 1}\right)$; though there are no data for cyclic 6-membered systems, linear selenenylsulfides are ca. $10^{4}$ times as electrophilic to selenolate attack at
Se as disulfides are at $S .{ }^{39}$ (ii) In general, selenolates are ca. 100 times as nucleophilic as thiolates. ${ }^{39}$ (iii) Selenols are also $2-3$ pKa units more acidic than thiols, ${ }^{34}$ and the acidity of $\mathrm{RSeH}$ in TrxR is still greater ${ }^{40}$. This enhances the general reactivity of the TrxR selenolate as compared to thiol species that are more likely to be protonated. Taken together, this suggests good initial rates of exchange at Se between TrxR and a cyclic selenenylsulfide. Importantly too, (iv) nucleophilic attack on the selenenylsulfide at Se is kinetically much more favoured than at $S,{ }^{41}$ fixing the initial attack site as the selenium.

(2): A selenenylsulfide's exchange intermediate should have the attacking nucleophile bound to Se, not to $S$ : The initial kinetic products of exchange with symmetrical cyclic dichalcogenides can rearrange intramolecularly within milliseconds to form the thermodynamic products. ${ }^{34}$ However, the thermodynamic preference for exchange intermediates to be Se-bound rather than S-bound is strong, regardless of the attacking nucleophile. ${ }^{42}$ For selenolate attack, a proximally $\mathrm{N}$-acylated linear diselenide intermediate has $\mathrm{E}^{\circ}$ ca. $270 \mathrm{mV}$ lower than the alternative linear selenenylsulfide (ca. $-550 \mathrm{mV}^{40}$ vs $-280 \mathrm{mV}$ ): so the diselenide should be the favoured intermediate (e.g. B rather than $\mathbf{E}$ in Fig. S4a). For thiolate attack, linear selenenylsulfides have $\mathrm{E}^{\circ} \mathrm{ca} .70 \mathrm{mV}$ lower than the alternative disulfide (ca. $-280 \mathrm{mV}$ vs $-210 \mathrm{mV}$ ), ${ }^{43}$ also favouring the Se-bound intermediate (e.g. D rather than $\mathbf{F}$ in Fig. S4a). Thus, the kinetically-favoured attack at Se (see (1)) also gives the thermodynamic intermediates, and these are the only intermediates relevant to probe evolution (B and $\mathbf{D}$ for probes of type $\mathbf{A} ; \mathbf{H}$ and $\mathbf{K}$ for probes of type $\mathbf{G}$; Fig. 2ab): which simplifies the pathways to cargo release (c.f. Fig. S4).

(3): A should avoid cargo release before reduction, and so have monothiol resistance: $\mathrm{The} \mathrm{E}^{\circ}$ values for the intermediates can be compared to the ca. $-364 \mathrm{mV}$ for intact cyclic selenenylsulfide triggers from Iwaoka's elegant study (Fig. S1d $).{ }^{42}$ Thus, the stability of linear diselenide intermediates $\mathbf{B}$ and $\mathbf{H}$ (from TrxR selenol attack; $-550 \mathrm{mV}$ ) better offsets the energetic penalty of opening the cyclic selenenylsulfide, than can linear selenenylsulfide intermediates $\mathbf{D}$ and $\mathbf{K}$ (from thiol attack; ca. $-280 \mathrm{mV}$ ). Thus, attack by TrxR should be much more thermodynamically favoured than by thiols.

Still, we anticipated that some thiol-exchange of $\mathbf{A}$ to $\mathbf{D}$ is unavoidable, particularly with monothiols due to their high cellular concentrations (Fig. 2c), so it is important to assess the fate of $\mathbf{D}$. We estimated that rates of cargo release directly from $\mathbf{D}$ (or $\mathbf{B}$ ) by 6-exo-trig "on-reductant" cyclisation of its thiol should be insignificant, particularly compared to those for 5-exo-trig cyclisation of the more acidic and more nucleophilic selenolate in fully reduced $\mathbf{C}$. Thus, we expected that $\mathbf{A}$ will significantly release cargo only if fully reduced to C. TrxR completes this reduction in a preorganised unimolecular step $(\mathbf{B} \rightarrow \mathbf{C})$, whereas monothiols would require collision and reaction with a second equivalent of RSH before fast intramolecular reclosure $^{34}$ returns A. Thus we also expected a very strong kinetic advantage for the rate of TrxR-induced rather than monothiol reduction to $\mathbf{C}$, which could combine to give overall monothiol resistance.

In contrast, exchange of regioisomer $\mathbf{G}$ to give $\mathbf{K}$ does allow the thiol intermediate to undergo fast 5-exo-trig cyclisation, permitting "on-reductant release" ( $\mathbf{k}_{\text {rel-k}}$ ) without full reduction to $\mathbf{J}$. Therefore, although the thermodynamics and kinetics of overall reduction of the trigger motifs of $\boldsymbol{A}$ and $\boldsymbol{G}$ are likely to be almost indistinguishable, we expect that probes of type $\mathbf{G}$ will suffer significantly more unwanted cargo release after unavoidable initial exchange with the monothiol background. (Though $\mathbf{H}$ can also give on-protein release $\mathrm{k}_{\mathrm{rel}-\mathrm{H}}$, full reduction to $\mathbf{J}$ by the preorganised TrxR is likely to be much faster 
than cyclisation, so we expect the $\mathrm{k}_{\text {rel-H }}$ pathway to play little role in TrxR-based cargo release: see section "Oxidoreductase profiling").

(4): Cargo release after reduction: Reduced $\mathbf{C}$ or $\mathbf{J}$ are small flexible reductants ${ }^{42}$ that are likely to reduce accessible disulfides in their environment $(\mathbf{C} \longrightarrow \mathbf{D})$ instead of cyclising to release cargo. Still, as $\mathbf{C}$ (selenolate) is likely to cyclise faster than $\mathbf{J}$ (thiol), we anticipated that $\mathbf{A}$ could be better than $\mathbf{G}$ for signal generation after full reduction in the cellular setting, where disulfides offer a return pathway.

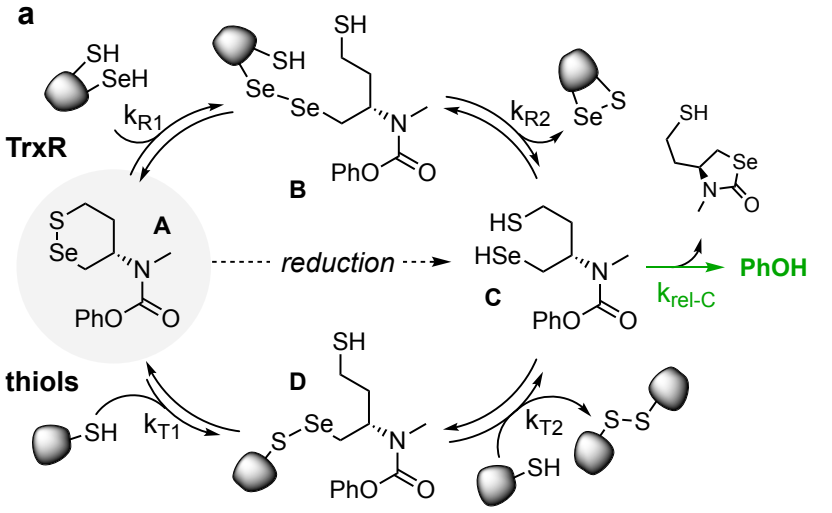

Taken together, these four aspects informed our hypotheses that probes of type $\mathbf{A}$ (which includes the $\mathbf{R X 1}$ probe) will be: (1-2) rapidly attacked by TrxR, (2) much more efficiently reduced through to C by TrxR than by thiols, (3) resistant to on-reductant signal generation by (mono)thiols, and (4) rapidly cyclise after reduction to $\mathbf{C}$. In contrast, though probes of type $\mathbf{G}$ will share features (1-2), they (3) should be labile to thiols: thus acting as a stringent control for our mechanism-based design.

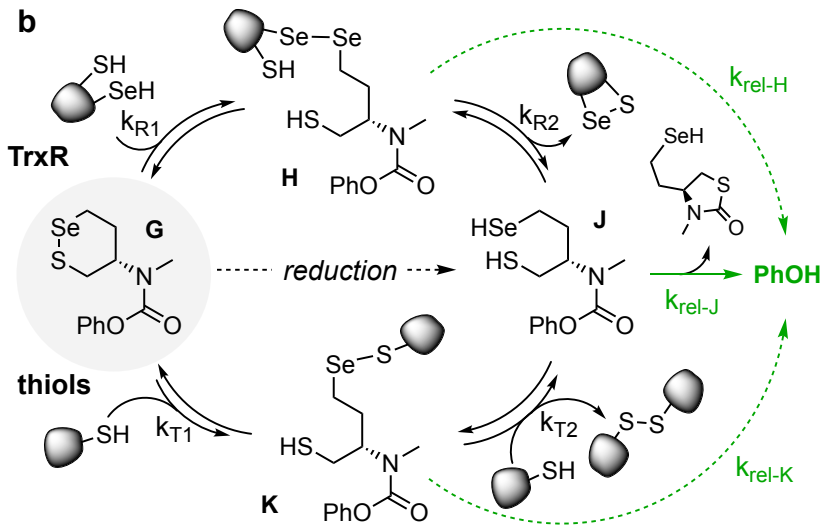

C key players in mammalian dithiol/disulfide chemistry
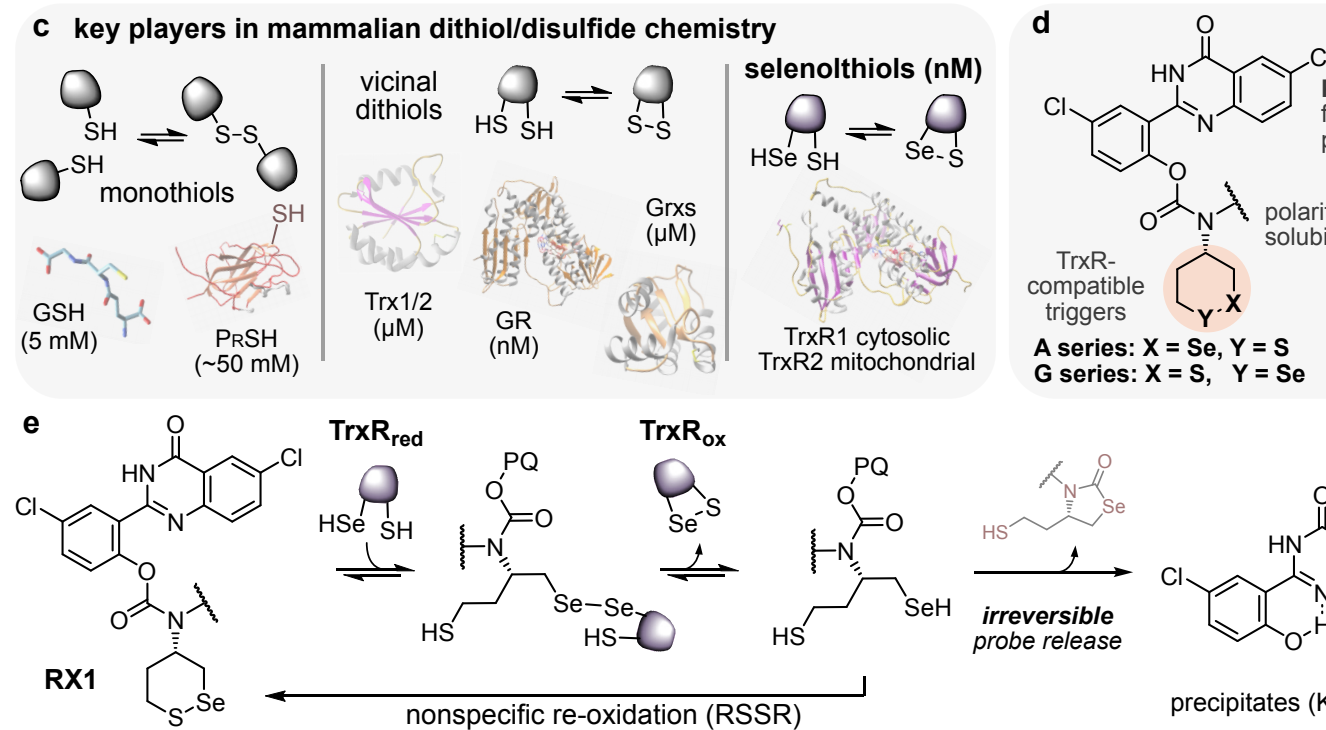

TrxR2 mitochondria

G series: $X=S, Y=S e$
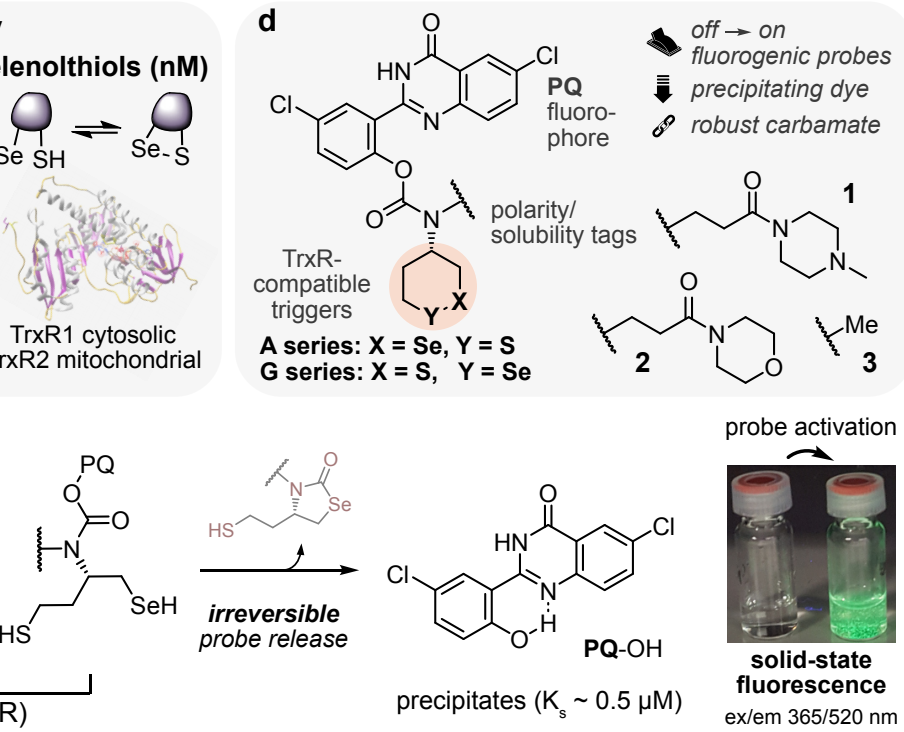

Fig. 2 Selenenylsulfides A, designed for TrxR-selectivity, and thermodynamically identical regioisomers G, designed for nonselectivity. a,b, Probes of type A (includes RX1) are predicted to avoid monothiol-based signal generation, whereas regioisomeric control probes of type $\mathbf{G}$ are predicted to be thiol-labile (full mechanism in Fig. S4). c, Some chemocompatible cellular reductants that a TrxR-selective probe must resist. d, Modular trigger-cargo design for fluorogenic probes with freely adjustable polarity. e, Signal activation pathway for RX1.

Probe design. We designed selenenylsulfide-based probes that use carbamate cyclisation to release phenolic cargos (Fig. 2d). This is a modular system that can be applied to any phenol-type cargos, including drugs. The phenolic carbamate system offers a range of desirable features, reviewed elsewhere $;^{17,44}$ important aspects are: (i) phenolates are good leaving-groups, giving fast cargo release, that is also orders of magnitude faster ${ }^{17}$ than with aniline carbamates that are more often ${ }^{36}$ used. (ii) Though primary amine phenolic carbamates are so unstable due to $\mathrm{E}_{1 \mathrm{cB}}$ elimination that they are usually discarded $^{36}$ (halflife $\sim$ seconds $^{45}$ ), secondary amine carbamates avoid $\mathrm{E}_{1 \mathrm{c}}$ and such probes are very robust to spontaneous hydrolysis (only ca. $1 \%$ in 3 weeks). ${ }^{44}$ (iii) Masking the hydroxyl of many phenols blocks their activities; so this system allows true off-to-on performance (zero activity before release) with a range of cargos. For example, phenol $O$-unmasking is required for activation of masked fluoresceins, ${ }^{17}$ indigo-type chromophores ${ }^{46}$ luciferins,${ }^{47}$ and bioactivity in many series of drugs (irinotecan ${ }^{48}$, duocarmycin ${ }^{49}$ ). Thus, it should be possible to extend this work's modular design to probes with diverse imaging modalities, and to many prodrug types.

We selected Haugland's precipitating fluorophore PQ-OH (Fig. 2d-e) $)^{50}$ as our proof-of-concept fluorogenic phenolic cargo. Acylating its phenol completely mechanistically quenches its highquantum-yield, environment-independent, large-Stokes-shift fluorescence (ex/em 360/520 nm), since this depends on excited state intramolecular proton transfer (ESIPT) of the phenolic hydrogen. Therefore PQ-releasing probes can operate as true turn-on systems, with zero self-background and excellent signal-to-noise ratios, typically above 300 even without background signal subtraction. There 
are many further advantages ${ }^{51}$ and few drawbacks of PQ-OH for cellfree and cellular proof-of-concept studies (see Supporting Note 4).

PQ probes with $\mathrm{N}$-methylated triggers proved to have low solubility and irreproducible results above $25 \mu \mathrm{M}$ in aqueous buffer with $1 \%$ DMSO. To address the challenge of the flat hydrophobic PQ without switching cargos, we used a basic $N$-methylpiperazinamide and a neutral morpholinamide as solubilising $N$-alkyl sidechains, to minimally crowd the redox-active site while being tracelessly removed upon cyclisation (Fig. 2d). The reproducibility of the resulting probes was greatly improved by their solubility, so we denoted the sidechains as 1 (piperazinamide, preferred; reliable to $>250 \mu \mathrm{M}$ ), 2 (morpholinamide), or 3 (methyl).

The modularity of this design ensures that any trigger can be fitted with any solubilising sidechain and any phenolic cargo. We freely combined triggers and sidechains to create PQ-releasing-probes, which we name by the letter of the trigger (e.g. A) combined with the number of the sidechain (e.g. 2), thus e.g. A2.

As well as the A-type selenenylsulfides and their G-type design controls, we designed hydrolytic, kinetic/thermodynamic, and mechanistic controls: reaching a panel of eleven cyclic probes and one linear probe (Fig. S5). We used an isosteric cyclohexane "trigger" as a non-reducible control for spontaneous or enzymatic hydrolysis, or aminolysis/thiolysis, of the carbamate (C-type); this is important when applying $\mathrm{mM}$ concentrations of thiolates (that might intermolecularly attack carbamates, confounding the desired reduction). We used a cyclic disulfide (S-type) and a cyclic diselenide (Se3) as reducible controls with different thermodynamic and kinetic sensitivity profiles, that we anticipated would make them not TrxR-selective. Linear disulfide $\mathbf{S S O O}^{23}$ (Fig. S5) was a mechanistic control, since according to our hypotheses it ought to access the irreversible pathway and so be thiol-labile (see Supporting Note 2).
Synthesis: We prepared the key selenenylsulfide building blocks in a straightforward, divergent manner from bis-mesylate $\mathbf{1}$ (Fig. 3a), which can easily be accessed from aspartic acid. ${ }^{52}$ Reaction with one equivalent of potassium thioacetate afforded a mixture of mono-substitution products $\mathbf{2}$ and 3, with a small preference for $\mathbf{2}$. These regioisomers were separated by chromatography. Treatment with potassium selenocyanate followed by thioester cleavage directly resulted in the formation of the cyclic dichalcogenides $\mathbf{5}$ and $\mathbf{8}$ by expulsion of cyanide. Boc-deprotection then gave the amine hydrochlorides 6 and 9. In parallel to our work, Iwaoka elegantly reported the synthesis of compounds $\mathbf{6}$ and $\mathbf{9}$ via a similar approach, but using non-commercial $\mathrm{PMBSeH}$ as the selenium source. ${ }^{42}$ In comparison, our synthesis offers more atom economy, reduced step count, fewer chromatographic separations and more rapid access to the final compounds, although these differences are not crucial. To obtain the control $\mathbf{S e}$ and $\mathbf{S}$ series dichalcogenides (Fig. 3a), bis-mesylate 1 was either treated with excess potassium thioacetate ${ }^{52}$ and $\mathrm{KOH}$ /air, or excess $\mathrm{KSeCN}$ and $\mathrm{KOH}$, followed by Boc-deprotection to form disulfide $\mathbf{1 0}$ or diselenide 11, respectively. The symmetrical linear disulfide $\mathbf{S S O 0}$ was accessed from commercial cystamine. We diversified these trigger building blocks by installing the sidechains, giving secondary amines. Finally, deprotection and coupling with chloroformate PQ-OCOCl ${ }^{44}$ (Fig. $\mathbf{3 b}$ ) yielded a panel of twelve PQprobes (Fig. 3c and Fig. S5): the A-type and G-type cyclic selenenylsulfides RX1, A2-A3 and G1-G3 as candidates/controls for TrxR-selective probes; the cyclic diselenide Se 3 and cyclic disulfides S1-S3 as well as the linear disulfide SSOO as reduction-responsive controls; and the non-reducible cyclohexyl probe $\mathbf{C 1}$ as a control for non-reductive activation. All probes were nonfluorescent as solids and in solution (see Supporting Information).

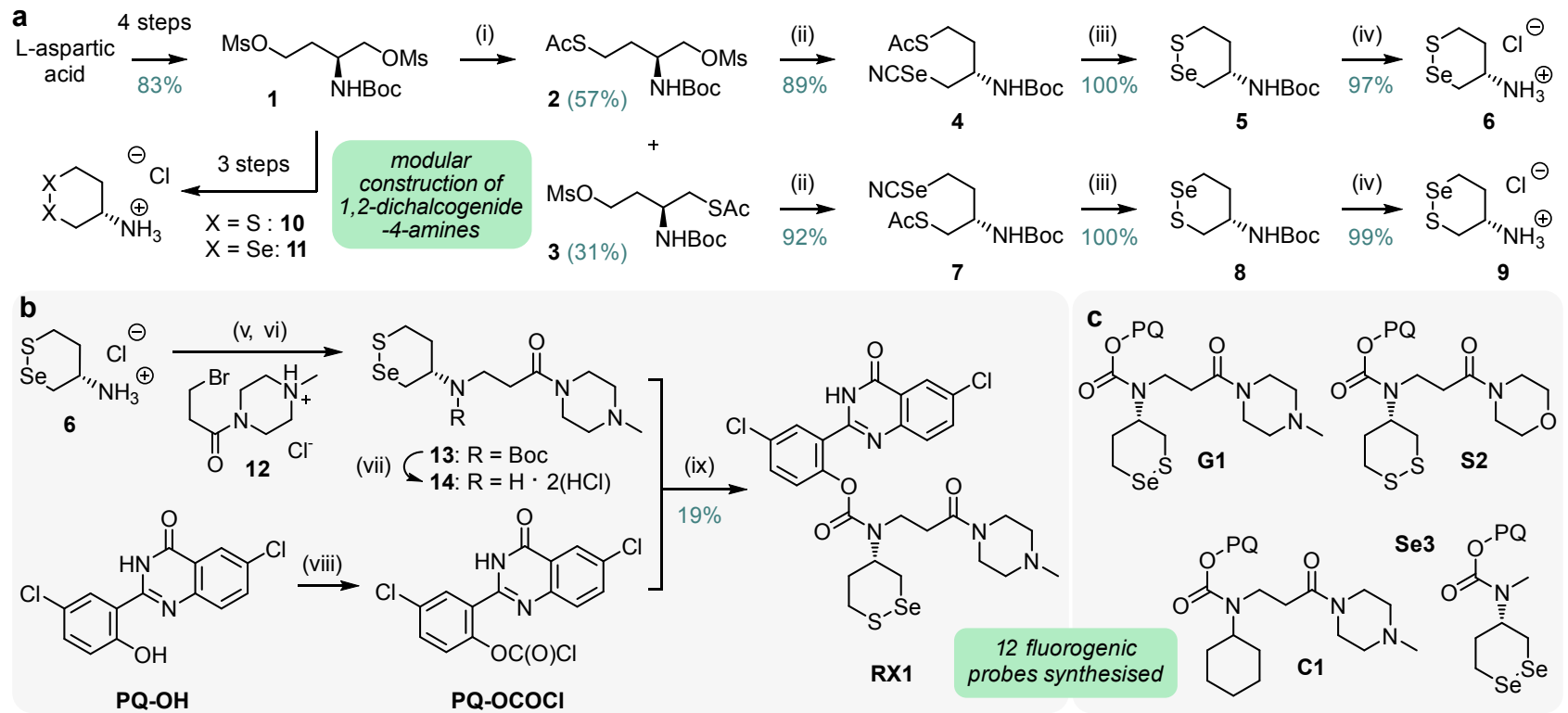

Fig. 3 Synthesis of triggers and probes. a, Divergent synthesis of amino-1,2-thiaselenane triggers: (i) KSAc, 18-crown-6, DMF, 15 h, r.t.; (ii) NaI, 18-crown-6, KSeCN, THF, 10 h, 50 ${ }^{\circ} \mathrm{C}$; (iii) $\mathrm{KOH}, \mathrm{MeOH} / \mathrm{THF}, 1 \mathrm{~min}$, r.t.; (iv) HCl, dioxane/DCM, 11 h, r.t.. b, Representative probe synthesis, shown for RX1: (v) $\mathrm{NEt}_{3}, \mathrm{MeOH}, 1 \mathrm{~h}, \mathrm{MW} 120^{\circ} \mathrm{C}$; (vi) Boc $\mathrm{B}_{2} \mathrm{O} \mathrm{NEt}_{3}, \mathrm{DCM}, 15$ h, r.t.; (vii) HCl, dioxane/DCM, 11 h, r.t.; (viii) triphosgene, DIPEA, DCM, 2 h, $0^{\circ} \mathrm{C} \rightarrow$ r.t.; (ix) DIPEA, DCM, 4 h, $0^{\circ} \mathrm{C} \rightarrow$ r.t. c, Representative PQprobes (full list in Fig. S5).

Cargo release mechanistic aspects. To test the 5-exo-trig cyclisation mechanism of Fig. 2a-b, we performed an HPLC-MS study of TCEP-mediated probe activation. This is critical since alternative post-reductive release mechanisms could give different kinetics and selectivity. For example, 3-exo-tet cyclisation releasing an $\mathrm{N}$-methyl probe carbamate via thiirane or selenirane formation (similarly to a 
report by Melnyk ${ }^{53}$ with halflife ca. $4.5 \mathrm{~h}$ ), followed by fast $\mathrm{E} 1_{\mathrm{cb}}$, could also be postulated as a mechanism. We also wished to test whether the triggers resist parasitic reactions such as TCEP deselenisation $^{54}$ which would otherwise complicate cell-free benchmarking.

In the TCEP challenge, $\mathbf{R X 1}$ reacted fully to selenocarbamate and PQ-OH. G1 reacted similarly although more slowly to give the thiocarbamate and PQ-OH, potentially reflecting the desired difference of $\mathbf{C} / \mathbf{J}$ cyclisation kinetics (section "Design", aspect 4). The 5rather than 6-exo-trig cyclisation is supported in two ways: (i) The RX1 cyclisation byproduct was found as the monomer, while the G1 byproduct was a monooxidised dimer matching interpretation as an easily-formed and highly stable linear diselenide. (ii) Equimolar selenolate alkylators almost entirely blocked PQ-OH release from RX1, but only slightly reduced PQ-OH release from $\mathbf{G 1}$. This latter observation crucially suggests that on-reductant cyclisation (which also proceeds from an intermediate with an engaged selenium) may be significant for $\mathbf{G 1}$ but not RX1: so matching design aspect 3. The controls ran as expected: SSO0 reacted fully to thiocarbamate and PQ-OH, while $\mathbf{C 1}$ remained intact. For full discussion see Supporting Information section 5.

In summary, the HPLC studies showed that reductive release is fast and operates by 5-exo-trig cyclisation according to the proposed mechanism; that deselenisation is not a complicating factor; and indicated that on-reductant cyclisation may be speedy (G1) or absent (RX1). As on-reductant cyclisation can be key for probes to resist (RX1), or succumb to (G1), monothiol-exchange-based triggering, we expected this to determine their reduction selectivity in cells.

Cell-free reductant profiling. To report on a protein selectively in cellular settings, a trigger must be completely resistant to reduction by the $\mathrm{mM}$ cellular monothiol background. ${ }^{18}$ Resistance or sensitivity to a reductant is best addressed by dose-response functions over time. Therefore, to best characterise the response to reductant challenges, we titrated reductants up to supraphysiological concentrations, with full timecourse rather than endpoint data. ${ }^{17,23}$

We began with GSH challenge tests. Over $6 \mathrm{~h}$, A-type probes showed zero fluorescence response to $10 \mathrm{mM} \mathrm{GSH}$, indicating outstanding monothiol resistance (Fig. 4a, Fig. S9-S10). The thermodynamically identical G-type controls were GSH-labile (3 mM GSH activated to ca. 25\%, Fig. S9-S10), supporting our pathway-based design (Fig. 2b). Dose-response plots of GSH resistance with the $6 \mathrm{~h}$ timepoint data (Fig. 4b, Fig. S10) highlight the GSHrobustness of all A-type probes and the partial GSH-lability of Gtype pathway controls. The comparison probe types behaved as expected. We had hoped that cyclic disulfides S1-S2, analogously to previously reported vicinal-dithiol-selective probe $\mathbf{S} 3,{ }^{17}$ would be monothiol resistant (i.e. no destabilisation by the solubiliser sidechains); they indeed resisted GSH up to $10 \mathrm{mM}$ (Fig. S9-S10). In comparison to these, linear disulfide $\mathbf{S S O 0}$ is a mechanistic control for the need of a cyclic dichalcogenide topology (Fig. 1c); its sensitivity to monothiol-based release was striking (nearly full activation by 1 mM GSH; Fig. 4a, Fig. S9). The cyclic diselenide Se3 was nonreactive (Fig. S9), suggesting that selenium is tolerated at both positions of the reduction-based probes without Se-oxidation-mediated release mechanisms (see Supporting Note 2). The zero-level fluorescence seen with RX1 (and Se3) under a thousandfold challenge by GSH also qualifies the secondary amine phenolic carbamate design as exceptionally stable to monothiolysis, hydrolysis and aminolysis.
Cell-free oxidoreductase profiling. We next tested the probes against a range of purified recombinant reducing enzymes in cell-free assays, including TrxRs as well as non-targeted Trxs, Grxs, GR, TRP14, and GPx1. The source and production methods are relevant for TrxRs, since the key selenocysteine (U498) residue in their active sites that is predominant in native enzymes, ${ }^{55}$ is typically not incorporated using standard recombinant expression systems. ${ }^{56}$ Therefore, we used human cytosolic TrxR1 and human mitochondrial TrxR2 produced with recent recombinant production methodologies that approach $100 \%$ CU active site content. ${ }^{57}$ To stringently test the validity of our design for selenolthiol-dependent activation, we also used the U498C point mutant of rat TrxR1 ( $\left.\operatorname{TrxR} 1^{\mathrm{U} 498 \mathrm{C}}\right) .^{58}$

The selenenylsulfide probes showed strong signal responses to TrxR1. RX1 at $10 \mu \mathrm{M}$ was strongly activated by $20 \mathrm{nM}$ TrxR1, with halftime to maximal signal of ca. $30 \mathrm{~min}$ (Fig. 4c): contrasting with its total stability to 1 million times higher concentrations of GSH over 6 hours. RX1 responded well to TrxR1 down to the subphysiological concentration of $5 \mathrm{nM}$ (Fig. 4d). As this cell-free assay underestimates probe sensitivity to low concentrations of TrxR (see Supporting Note 5), this was encouraging for cellular use. TrxR1turn-on kinetics and response profiles were similarly good for all other cyclic selenenylsulfide probes (A2-A3, G1-G3) (Fig. S13). This unity of TrxR1-selective performance despite the structural diversity of the probes matched our hypothesis that the cyclic selenenylsulfide is responsible for reactivity, and that the solubilising motif can be exchanged without altering the triggers' redox activity. An important result for selectivity, which completes the $\mathrm{k}_{\text {rel-H }}$ discussion (see section "Design"), is that the rates of probe activation by TrxR1 were essentially identical between the A-type and $\mathbf{G}$-type triggers (Fig. S13). If on-TrxR cyclisation (Fig. 2a-b) were significant for probe release, the G-type probes would be significantly faster than the A-type in releasing cargo. However, the data rather support that TrxR1 indeed fully reduces the cyclic selenenylsulfide probes to $\mathbf{C} / \mathbf{J}$, before they cyclise: full reduction by $\operatorname{TrxR}$ is faster than cyclisation.

Disulfide controls S1-S3 were nearly inert to TrxR1 (Fig. S13); though piperazinamide sidechain 1 gave faster reaction speeds compared to less soluble methyl and morpholinamide sidechains, presumably due to hydrophobic adsorptive loss ${ }^{23}$ of the intact probes as well as local $\mathrm{pH}$ modulation. This effect was often seen later with Aand G-type probes, and supported the choice of piperazinamides as the standard sidechain for the probes. Diselenide control Se3 was completely nonresponsive to TrxR1, as it later proved also to be to all other reductants except to DTT (Fig. S9, Fig. S12).

Of the selenenylsulfides, only RX1 and $\mathbf{G 1}$ were reduced by mitochondrial TrxR2, matching the trend of greater reactivity for the piperazinamides, and only at high TrxR2 (50 - 100 nM; Fig. 4c, Fig. S13). Some TrxR2 response was expected, as it has the same Sec-Cys motif as TrxR1, but this mirrors ample literature precedents of TrxR2 being slower to process small molecule substrates than TrxR1. ${ }^{59}$ Since cytosolic TrxR1 has many-fold higher total expression in cells, we therefore expected the faster-reactive TrxR1 to be the dominant cellular reductant for our selenenylsulfide probes.

Next, we wished to test our hypothesis that the selenenylsulfides should interact selectively with mammalian TrxR on the basis of the TrxR selenolthiol. TrxR has two redox-active sites, with the $\mathrm{NADPH}$-driven N-terminal Cys-Cys shuttling electrons to the Cterminal Sec-Cys. As Hondal et al have shown in their paper "No Selenium Required", 32 many compounds that had been assumed to require a selenol for reactivity can be similarly processed by a C- 
terminal Cys-Cys mutant $\operatorname{TrxR}^{\mathrm{U} 498 \mathrm{C}}$, or are better or exclusively processed by the $\mathrm{N}$-terminal dithiol site. We compared probe activation by wildtype TrxR (Sec-Cys) with Cys-Cys mutant $\mathrm{rTrxR} 1^{\mathrm{U} 498 \mathrm{C}}$. The mutant no longer reduced either the A- or G-type probes, while its reduction of standard substrates DTNB and juglone was unimpeded (Fig. 4e, Fig. S14). This supported that both A- and G- selenenylsulfide probes do indeed require native mammalian TrxR's C-terminal selenolthiol for efficient reduction, matching our designs.
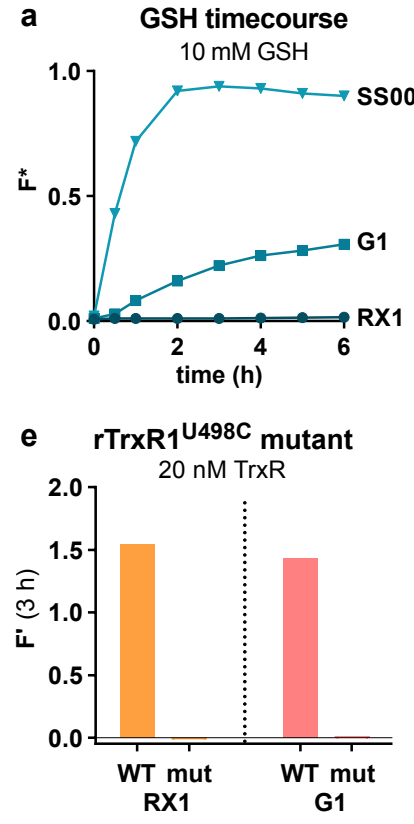

b GSH dose-response

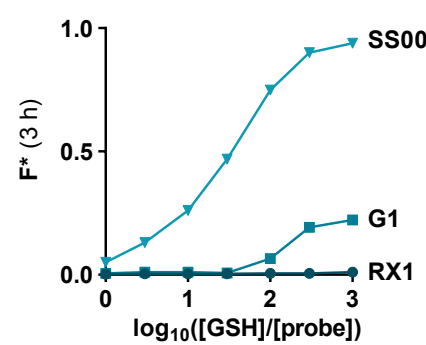

f Trx/TrxR activation of RX1

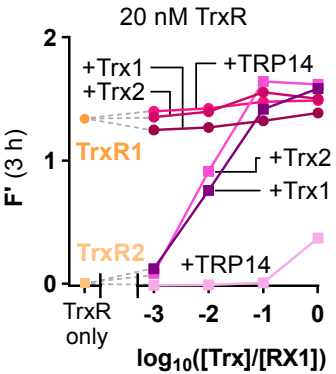

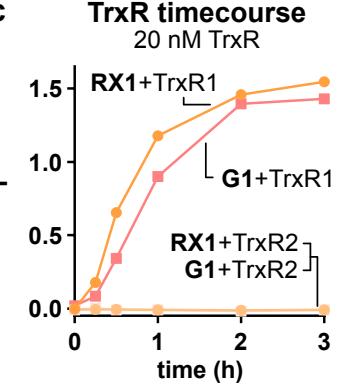

g Trx1 dose-response

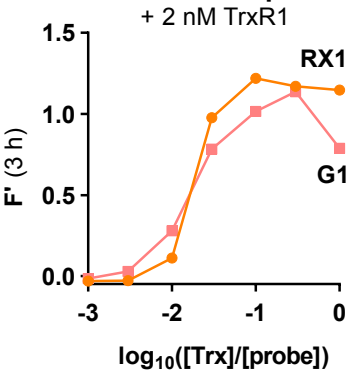

d TrxR1 dose-response
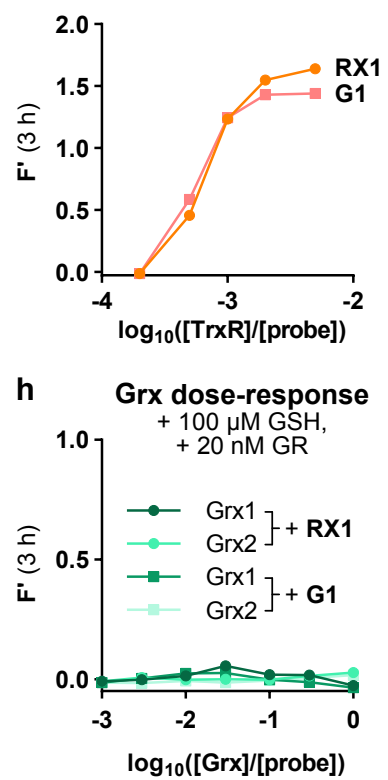

Fig. 4 | In vitro characterisation of TrxR-selective RX1 vs comparison probes. a,b, Selected GSH-resistance timecourses, and GSH-dose-response (0.01-10 mM GSH). c, Selected TrxR1-activation timecourses (20 nM TrxR1 and TrxR2). d, TrxR1-dose-response. e, Selenenylsulfide probe activation by wildtype TrxR (Sec-Cys) compared to Cys-Cys mutant $\mathrm{rTrxR}^{\mathrm{U} 498 \mathrm{C}}$. f, Dose-response titrations with vicinal dithiols Trx1, Trx2, and TRP14 (0.01-10 $\mu \mathrm{M})$ in presence of $20 \mathrm{nM}$ TrxR. $\mathbf{g}$, Trxl dose-response (0.01-10 $\mu \mathrm{M})$ titrations in the presence of $2 \mathrm{nM}$ TrxR. h, Dose-response $(0.01-10 \mu \mathrm{M})$ profiles to the GR/GSH/Grx cascade $(20 \mathrm{nM} \mathrm{GR,} 100 \mu \mathrm{M}$ GSH). [All probes used at $10 \mu \mathrm{M}$ in TE-buffer. TCEP (10 equiv., $100 \mu \mathrm{M})$ was used as a reference for fast, quantitative probe reduction and activation; $\mathbf{F}^{*}$ in enzyme-free assays is the relative fluorescence signal compared to full activation $\left(\mathbf{F}^{*}=\mathrm{F} / \mathrm{F}^{\mathrm{TCEP}}\right)$. NADPH $(200 \mu \mathrm{M})$ was applied as native upstream component for enzymatic assays; $\mathbf{F}^{\prime}$ in enzymatic assays is $\mathrm{F}^{*}$ additionally corrected for NADPH autofluorescence (see Supporting Information).]

With excellent monothiol resistance, and strong processing by selenolthiol TrxR1 rather than its dithiol mutant, it remained an open question whether physiological concentrations of other selenolbearing proteins would also be capable of probe triggering, which is important for enzymatic selectivity. There are only 25 selenoproteins in the human proteome; only a few of these have ubiquitous expression at substantial concentrations; and nearly all of those have monoselenol active sites (not selenolthiols). The highest-concentration ubiquitous selenoproteins are monoselenol glutathione peroxidases GPx1 and GPx4. ${ }^{10}$ We challenged RX1 and G1 with GPx1 up to supraphysiological $1 \mu \mathrm{M}$ concentration, and no probe activation was detected over 6 hours (Fig. S15). This was an encouraging step towards the possibility of cyclic selenenylsulfides acting as TrxR-selective probes (see Supporting Note 6).

Having tested the major biological monothiol, monoselenol, and selenolthiol reductants, we finally examined the effect of vicinal dithiols on probe activation. We began by screening the probes' doseresponse to dithiothreitol (DTT), a nonphysiological vicinal dithiol that is ca. $50 \mathrm{mV}$ more reducing than Trxl, but which reproduces some aspects of its behaviour and so can be helpful to study dithiol reactivity. ${ }^{17}$ Excess DTT triggered all A- and G-type selenenylsulfide probes. Although selenenylsulfides are more thermodynamically resistant to reduction than disulfides, their reaction with DTT was more rapid and more sensitive than that of the corresponding S1-S3 series (Fig. S11). This is a reminder of the importance of kinetics to probe release, as the greater electrophilicity of Se in selenenylsulfides than $S$ in disulfides determined the assay outcomes.

We then tested Trxs, titrating them to physiological $10 \mu \mathrm{M}$ in the presence of $10 \mu \mathrm{M}$ probe. These assays require TrxR and NADPH to reduce Trx from the non-reactive, oxidised, stored state, and to rereduce it after each oxidation by probe or by molecular $\mathrm{O}_{2}$ in the non-degassed assay buffer. RX1 is already fully and rapidly activated by $20 \mathrm{nM}$ TrxR1 alone (Fig. 4c), so, unsurprisingly, titrating in Trx1/2 or other Trx-fold proteins such as TRP14 did not reveal extra processing by those species (cf. TrxR1 data in Fig. 4f). To study direct probe reduction by Trx only, we instead used concentrations of TrxR1 and TrxR2 that were ineffective at directly activating RX1, observing half-maximal probe activation with ca. 0.1-0.2 $\mu \mathrm{M}$ of Trx1 or Trx2 regardless of which TrxR was chosen (TrxR2 data in Fig. 4f, Fig. S17 and Fig. S19; TrxR1 data in Fig. 4g and Fig. S22). This represents a ca. 50-fold lower sensitivity to Trx than to TrxR1, under continually re-reduced conditions. The TrxR-resistant disulfide comparison probes S1-S3 were activated by Trx as expected, ${ }^{17}$ with half-maximal activation around $0.3 \mu \mathrm{M} \operatorname{Trx}$ (Fig. S16-20).

We finally tested alternative redox-active dithiol proteins. We first profiled dose-response to Grx1 and Grx2, the other major vicinal dithiol protein redox effectors, applied as part of the full GR-GSH-Grx cascades (Fig. 4h, Fig. S23-S24). Both RX1 and G1 fully resisted up to $10 \mu \mathrm{M}$ Grx1/Grx2 over 3 hours (including $20 \mathrm{nM} \mathrm{GR,} 200 \mu \mathrm{M}$ $\mathrm{NADPH}$, and $100 \mu \mathrm{M}$ GSH for Grx reduction). This is a significant 
result. Although the Grxs are vicinal dithiol redox enzymes similar to the Trxs, they are more RSSG-selective. Therefore the cyclic probes should resist Grx processing inasmuch as they resist monothiol exchange (note too that although GR is NADPH-driven, it has very high specificity for GSSG so is not expected to directly reduce the probes). We also examined the TrxR-reduced redox-active vicinal dithiol TRP14, finding that the probes resisted it up to $3-10 \mu \mathrm{M}$ (Fig. 4f, Fig. S18, Fig. S21). These results show that the probes are not sensitive to all vicinal dithiol proteins per se, but have a specific sensitivity to the highly reducing Trxs at least in cell-free conditions. While it would have been best for our TrxR-reporter goal if Trxs were incapable of probe reduction, sensitivity can be expected in a cell-free assay where excess NADPH and an absence of other oxidants and binding partners offers no alternative pathway except probe reduction. In cells however, Trx as an effector protein has many binding partners that recognise and oxidise its thioredoxin fold, while NADPH-powered TrxR binds fewer substrates (mainly Trx, also TRP14, and potentially Grx2). ${ }^{13,60,61}$ This gave hope that if Trx in cells would be substantially engaged with preferred protein substrates, then RX1's extraordinary kinetics of turnover by TrxR1, combined with its total stability to GSH, might give it functional TrxR1-selectivity in cells. In line with this understanding, previous research on Trx has identified cyclic dichalcogenide probe chemotypes that were highly sensitive to purified $\operatorname{Trx}$ (but not to TrxR) in cell-free settings, yet were unexpectedly inactive in cellular settings despite the high cellular concentrations of this universal reductant. ${ }^{17}$
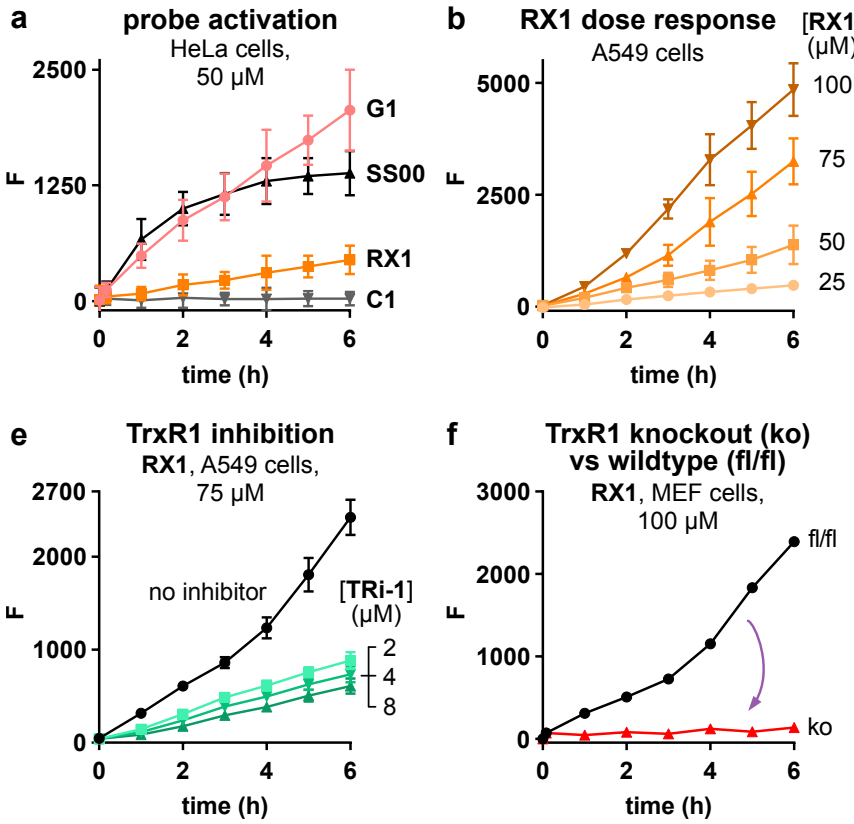

Cellular TrxR1-selectivity: By now we had shown that, in line with our pathway hypothesis, RX1 was monothiol resistant and could therefore be a protein-selective probe, while its regioisomeric control G1 had some lability to physiological GSH concentrations. Under cell-free conditions, both $\mathbf{R X 1}$ and $\mathbf{G 1}$ were rapidly activated by TrxR1, dependent upon its selenolthiol active site, and had minor sensitivity to TrxR2; both were sensitive to fully reduced Trx but almost entirely nonresponsive to the alternative vicinal dithiol thioredoxin-related protein TRP14; and were inert to GR/Grx. We now entered cell-based assays, aiming to test the probes' TrxR-dependency indicated in the cell-free assays as well as their potential for functional TrxR1-selectivity in the cellular context, by elucidating the cellular mechanism of activation of these compounds (see Supporting Information for assay conditions, processing methods, full data for all probes and accompanying discussions; and Supporting Note 5 for discussion of fluorescence normalisation).

Signal from the selenenylsulfide probes was strong in different cell lines (Fig. 5a-b), dose-dependent (Fig. 5b), stable over long timecourses, and not due to direct carbamate cleavage (C1, Fig. 5a). The potentially TrxR-selective RX1 was predictably slower to generate signal than nonselective disulfide SSO0 or GSH-sensitive G1. Fluorescence imaging showed near-ubiquitous turn-on of RX1 with PQ-OH fluorophore being cellularly retained for hours (Fig. 5d).

Fig. 5 Cellular evaluation of TrxR-selectivity of RX1. a,b, fluorescence activation in HeLa and A549 cell lines (three independent experiments, mean with SD). c, Cellular RX1 signal depends on selenium starvation/supplementation, while comparison probes SSO0 and G1 do not. d, Fluorescence microscopy images showing cell-marking performance of the PQprobes. Overlay of PQ-OH fluorescence (green) on brightfield images. e, Cellular RX1 signal is efficiently suppressed by the TrxR inhibitor, TRi-1. f,g, Genetic knockout of TrxR1 entirely suppresses signal generation by RX1, whereas reconstitution of TrxR1 expression restores RX1 activation (MEF [TrxR1 ${ }^{\mathrm{ko}} / \operatorname{TrxR} 1^{\mathrm{ki}}$ ] cell lines). [F is the raw fluorescence signal minus the signal of DMSO-only controls (platereader assay; 20,000 cells per well, three wells per datapoint); $\mathbf{F}$ ' is the raw signal minus its time-zero value, i.e. $\mathbf{F}^{\prime}\left(\mathrm{t}_{0}\right) \equiv 0$ by definition (hence time-zero is not shown in $\mathbf{c}$ ), which gives a more sensitive comparison of conditions; see Supporting Information)]

We then performed several orthogonal tests of whether cellular activation of the $\mathbf{R X 1}$ probe is indeed TrxR-selective.

Cells cultured without selenium supplementation do not fully incorporate Sec in TrxR but instead incorporate Cys, so lowering the cellular concentration of the selenolthiol form of $\operatorname{TrxR}{ }^{62} \mathbf{R X 1}$ signal was four times higher in cells supplemented with selenium than without (Fig. 5c), whereas SSO0 and G1 were essentially unaffected. This is consistent with an interpretation that RX1 activation 
in cells depends on native TrxR, while the mechanistic control G1 (just like nonselective SSO0) is not selective in the cellular context for TrxR (presumably, the residual GSH lability of G1 allows it to be activated by the monothiol background; Fig. 4b).

We next explored how chemical inhibition of TrxR affects signal generation. We relied on the metal-free compounds TRi-1 and TRi-3 which are inhibitors with good cellular TrxR-selectivity, ${ }^{63}$ rather than the common thiophilic gold complex auranofin which has potential pitfalls $^{23}$ (see Supporting Note 1 and Fig. S2). Cellular RX1 signal was strongly inhibited by acute TRi treatment, with just $2 \mu \mathrm{M}$ TRi-1 reducing signal by $66 \%$; whereas G1 signal was only reduced by $15 \%$ and SSO0 signal was unaffected by this treatment (Fig. 5e, Fig. S26). TRi-3 dose-dependently reproduced the same inhibitory effects. This supports that cellular RX1 activation is highly dependent on the activity of the TRi-1/3 target TrxR, whereas activation of its regioisomer $\mathbf{G} \mathbf{1}$ is largely TrxR-independent.

Finally, we stringently evaluated RX1's TrxR1-dependency using TrxR1-knockout (ko) cells (MEF: mouse embryonic fibroblasts) ${ }^{64}$, which we compared against their corresponding wildtype control $(\mathrm{fl} / \mathrm{fl})$. This knockout upregulates compensatory thiol-based reductive pathways to survive (e.g. the GSH system), with Trx being mainly reduced instead by Grx2. ${ }^{65,66}$ Therefore, we expected that the only circumstances under which we would see zero signal for a hitherto cellularly-active probe dependent on Se-containing TrxR1, would be if that probe was exclusively activated by TrxR1 in the cellular context. The TrxR1 ${ }^{\mathrm{ko}}$ MEFs showed zero RX1 activation, while the corresponding wildtype gave strong signal (Fig. 5f). As the mitochondrial TrxR2 is still functional in the knockout, this strongly suggests that cellular RX1 signal reports essentially on cytosolic TrxR1 at least in this cell line. Signal from mechanistic control G1 was suppressed by ca. $60 \%$ though not abolished in the knockout cells (Fig. S27a), again matching expectations that G1 is not fully TrxR1-selective in cells. To obtain still stronger proof of mechanism, we tested TrxR1-knock-in (ki) to the MEF TrxR1-knockout background $^{65}$. This fully restored the strong, dose-dependent RX1 signal generation (Fig. 5g, Fig. S27b).

Taken together, the data strongly support that RX1 is exclusively activated in cells by TrxR1. RX1 is therefore the first validated probe design that can selectively and meaningfully report on the activity of mammalian TrxR1 non-invasively in live cells.

RX1 enables quantitative high-throughput cellular screening for TrxR1 inhibitors: Inhibitors of TrxR1 hold promise as therapeutics for treating cancer, autoimmune and inflammatory diseases. The chalcophilic gold complex auranofin (Ridaura) is one effective though poorly selective TrxR inhibitor, clinically used against the autoimmune inflammatory disease, rheumatoid arthritis. It and many analogues complexes have reached late-stage clinical trials in cancer, motivated by tumoral upregulation and reliance upon redox systems. ${ }^{67}$ Other TrxR inhibitor classes include redox-active species and organic electrophiles. Until now, TrxR1 assays to guide inhibitor development were enzymatic ("cell-free") or utilised cell lysates. The technical and cost challenges of expressing and purifying mTrxR with near-quantitative selenium-incorporation on a sufficient scale for large screening, have limited enzymatic TrxR screening: only one high-throughput screen (HTS) has been reported. ${ }^{63,68}$ Enzymatic or lysate assay hits can be irrelevant in cells (poor permeability, biolocalisation, or target specificity), report artifactual hits (e.g. fluorescence quenching or aggregation), and can identify promiscuous compounds that are unlikely to be useful in biology, rather than selective compounds. Biochemical assays also cannot identify compounds that are biotransformed into active inhibitors: a field that is recently emerging for selenoprotein targeting. ${ }^{69}$

Cellular HTS can be far simpler and cheaper to perform; and allows screening different cell lines, towards therapeutic TrxR inhibitors effective in target cells (with varied expression levels of TrxR and of likely off-target thiol / selenol species), while controlling for drug-relevant performance issues such as upregulated electrophile detoxification and drug efflux pumping, and cell-type-dependent uptake. If a selective probe/readout is used, the data can also be more likely to emphasise selectivity over nonspecific reactivity, making it more actionable in drug development. A HTS-suitable probe must be TrxR-selective; but it must also pass many additional criteria for automatic operation, including (a) broad dynamic range (negligible background and cellular crosstalk, high signal-to-noise); (b) broad linear range (for quantitative use); (c) minimal count of error-prone steps (no additional reagents or handling); (d) no manual tailoring of conditions or processing by compound classes; (e) high-quality data: stable signal, with high precision (small deviations) and high accuracy (confidence of TrxR quantification, minimum interference from test compounds) (see Supporting Information).

The stability and constant environment of RX1's signal (crystallising fluorophore, protected from interferences), its low background (ESIPT quenched probe) and low crosstalk (high Stokes shift), and its ability to directly generate a readout, were promising features. We therefore set out to develop the first cellular quantitative HTS (qHTS) assay for TrxR1 inhibitors, using RX1, and performing pilot screening with the 1280-compound $\mathrm{LOPAC}_{1280} \mathrm{li}$ brary. ${ }^{70}$ LOPAC is intended to cover drugs and drug-like scaffolds with much comparative data on target selectivity, potency, cellular and in vivo bioactivity, without focus on any particular mechanism of action. The LOPAC library was used in the only previously reported qHTS enzymatic TrxR assay (in 2011), and there is $82 \%$ overlap in composition between the 2011 and 2021 versions. $^{68}$

Cellular screening using RX1, optimized for HTS with 1536-well plates (assay volume $6 \mu \mathrm{L}, 500$ cells/well) over $4 \mathrm{~h}$ run-time (Table S1), reproduced the strong performance seen in 96-well format (Fig. 4-5). Untreated vs no-cell controls gave a 7-fold raw signal to background ratio $[\mathrm{S}: \mathrm{B}]$ without background compensation, with a high Z' value of 0.64 , suitable for HTS (Fig. 6a). RX1 signal was linear over the assay time, and linearly reflected turnover rate (varied RX1 concentration or cell count; Fig. 6b). Pre-incubating cells for $1 \mathrm{~h}$ with reference inhibitors prior to RX1 gave concentration-dependent inhibition consistent with reported values (Fig. 6c).

A LOPAC ${ }_{1280}$ screen using this assay protocol performed well ( $Z^{\prime}$ $=0.63, \mathrm{~S}: \mathrm{B} 7: 1)$. Compounds with apparent toxicity within the assay time were excluded by a separate viability counter-assay. Moderate cut-off criteria $\left(\mathrm{IC}_{50}<20 \mu \mathrm{M}\right.$ and curve form; see Supporting Datafile) gave a $<1.5 \%$ hit rate ( 18 of 1278 compounds, plus TRi- 1 and TRi-2; Fig. 6d, Fig. S30). None of the LOPAC 1280 compounds were expected to have truly TrxR-selective inhibitory activity in cells: the panel serves to demonstrate HTS assay performance and likely hit rates in larger-scale screening, and to identify trends among hit classes. Pleasingly, the hit rate was manageably low, and the hits are indeed likely selenol-reactive species: 3 heavy metal complexes, 13 organic electrophiles or redox-active species, and a known TrxR-inhibiting porphyrin which may act via redox ${ }^{68}$ encouragingly, only one non-obvious hit was present, the glutamate receptor ligand AIDA (Fig. S30). pIC 50 values also followed likely cellular reactivity: no 
change despite ca. 1.1 LogP difference between lipophilic vinylsulfones BAY 11-7085 (methyl) and BAY 11-7082 (tert-butyl); but 10 -fold lower for permeability-limited drug aurothioglucose than its ester prodrug auranofin; etc (Fig. 6d, Fig. S30). Matching expectations, with the exception of auranofin, none of these species approached the potency of TRi- $1 .^{63}$

One opportunity afforded by RX1-enabled cellular screening is to compare, for the first time, cell-free to cellular TrxR inhibition by species, to assess trends for cellularly-useful or -less-useful inhibitors. 1047 LOPAC compounds were assessed across both the screens ${ }^{68}$, of which 1011 generated robust enough data quality for comparisons (Table S2; Supporting Information section 4.2). Of these, 993 compounds were inactive in both assays, and 7 were active in both. This $99 \%$ overlap of conclusive results between RX1 and the purified enzyme screen speaks strongly to the precision of the cellular RX1 assay. Only 2 compounds, IPA-3 and chloro-APB, were active in the cellular screen despite inactivity in the enzymatic assay: and these are catechol-like species with plausible redox activity in cells. To us, this indicates that the cellular RX1 assay has excellent robustness against false positives. Given that hits were classed up to $20 \mu \mathrm{M}$, while RX1 relies on precipitation of $>1 \mu \mathrm{M}$ of a flat aryl fluorophore for signal generation, we had feared false positive inhibition from the many LOPAC PAINS (pan-assay interference compounds) that have aggregation effects, or could alkylate the reduced intermediate C (Fig. 2a). However, not even powerful cellular PAINS rottlerin, rhodanine and myrcetin $^{71}$ gave apparent potencies above $3 \%$ of that of the reference inhibitor TRi-1 (Fig. 6d). Finally,
9 compounds were inactive in the RX1 assay despite activity in the enzymatic screen. These were also redox-actives and electrophiles (catechols, $\boldsymbol{\alpha}$-haloketone, nitrosyl donor, Michael acceptors, wortmannin), which may indicate filtering of low-quality hits by the more stringent cellular assay (Table S3 and Supporting Datafile).

Interestingly, the ratios of cell-free to cellular potencies of the shared hits clustered with their reactivity (Fig. 6e-f). The $S_{N} A r-r e-$ active species had near-identical cell-free and cellular potencies; heavy metals and nitrosylating agent Dephostatin had $>10$-fold potency loss in cells; and Michael system electrophiles up to $\sim 100$-fold potency loss. The on-target potency of irreversibly reactive compounds in cellular assays is strongly ruled by their biolocalisation and reaction rates with off-target species. We expect that our data comparison (Fig. 6f) reflects the degree of undesirable drug loss to offtargets according to compound class; and we propose that the superior cellular translation of the selenophilic tempered $S_{N} A r$ electrophiles suggests how drug development can pursue both effective and selective inhibitors of this key oxidoreductase.

In conclusion, these strong results give confidence that cellular qHTS with RX1 is a valid and valuable TrxR inhibitor screening strategy, promising that focused libraries can be used with cell lines of choice to guide development of cellularly-acting TrxR inhibitors. We suggest that tempered $S_{N} A r$ electrophiles would be a good starting-point for such developments. Certainly, quantitative, robust, and selective cellular TrxR probes, with the HTS-compatibility and high data quality that RX1 has shown across these assays, will be vital to guide progress in this new territory of redox-targeting drugs.
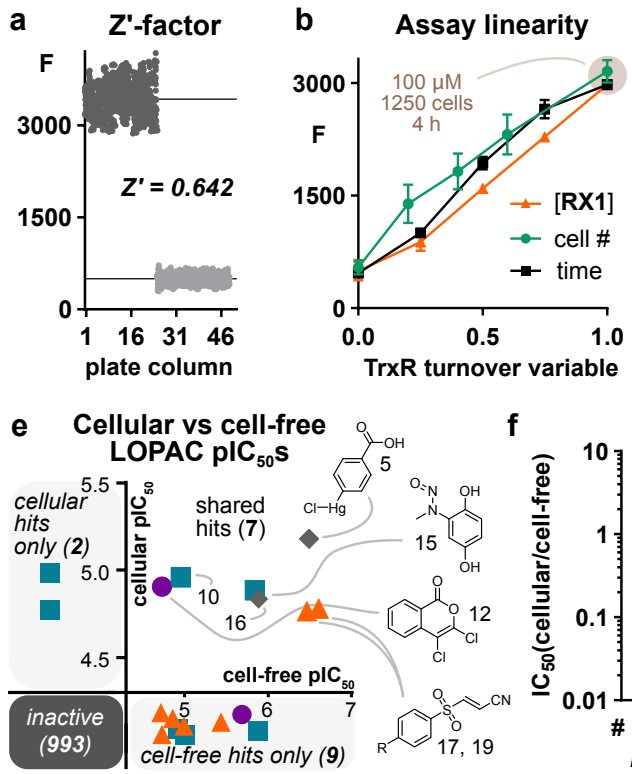$$
\text { f }
$$

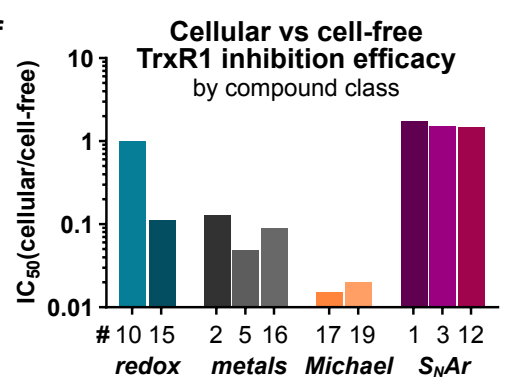

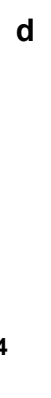

\begin{tabular}{|c|c|c|c|}
\hline \# & compound & $\mathrm{plC}_{50}$ & notes \\
\hline 1 & TRi-1 & 6.5 & E: Se-targeted $S_{N} A r$ \\
\hline 2 & Auranofin & 6.0 & $E:$ heavy metal \\
\hline 3 & TRi-2 & 5.6 & $E$ : Se-targeted $S_{N} A r$ \\
\hline 4 & Rottlerin & 5.3 & E': electrophile PAIN \\
\hline 5 & 4-ClHgBzOH & 5.2 & $E$ : heavy metal \\
\hline 6 & Myricetin & 5.1 & $\boldsymbol{R}^{\prime}$ : polyphenol PAIN \\
\hline 7 & AIDA & 5.1 & not classified \\
\hline 8 & IPA-3 & 5.0 & $\boldsymbol{R}$ : aryl disulfide \\
\hline 9 & TPCK & 5.0 & E: a-halocarbonyl \\
\hline 10 & Protoporphyrin IX & 5.0 & $R$ : redox \\
\hline 11 & AS604850 & 5.0 & $E / R$ ': rhodanine PAIN \\
\hline 12 & $3,4-\mathrm{Cl}_{2}-$ Coumarin & 4.9 & $E: \mathrm{S}_{N} A r$ reactivity \\
\hline 13 & Benserazide & 4.9 & $\boldsymbol{R}:$ redox \\
\hline 14 & Azathioprine & 4.9 & E/R: $\mathrm{S}_{\mathrm{N}} \mathrm{Ar} /$ redox \\
\hline 15 & Dephostatin & 4.9 & E/R: NO-donor / redo) \\
\hline 16 & Aurothioglucose & 4.8 & $\boldsymbol{E}$ : heavy metal \\
\hline 17 & Bay 11-7082 & 4.8 & $E$ : vinylsulfone \\
\hline 18 & Chloro-APB HBr & 4.8 & $R$ : redox \\
\hline 19 & Bay 11-7085 & 4.8 & $E$ : vinylsulfone \\
\hline 20 & Tyrphostin 23 & 4.8 & E: dicyanoalkene \\
\hline
\end{tabular}

Fig. 6 | A high-throughput screen with RX1 quantifies cellular TrxR inhibition by pharmaceutically active compounds. a, Wide assay dynamic range and excellent data precision lead to an outstanding $Z^{\prime}$ value (cells vs no cells controls). $\mathbf{b}$, The signal-turnover relationship of $\mathbf{R X 1}$ is linear over the whole dynamic range (parameters varied: incubation time, RX1 concentration, cell count; standard parameter values normalised to 1). $\mathbf{c}$, Doseresponse plots obtained in $6 \mu \mathrm{L}$ automatic RX1 HTS for reference inhibitors. d, Potencies for all 18 hits from LOPAC ${ }^{1280}$ RX1 qHTS plus TRi-1 and TRi-2, annotated for reactivity and compound class $\left(\boldsymbol{E}\right.$ : electrophile, $\boldsymbol{R}$ : redox-active; all structures in Fig. $\left.\mathbf{S 2 9} ; \mathrm{pIC}_{50}=-\log _{10}\left(\mathrm{IC} \mathrm{C}_{50}[\mathrm{M}]\right)\right)$. e, Potency distribution among compounds tested in current cellular as well as previous cell-free qHTS. f, Ratios of cellular to cell-free potencies of shared hits suggest that $\mathrm{S}_{\mathrm{N}} \mathrm{Ar}$-based TrxR-inhibitors may translate well through development.

\section{DISCUSSION}

TrxR's position as a central redox node in diverse physiological processes drives the hitherto-unmet need for precise, selective molecular probes to characterise and decrypt its activity in cell biology and in physiological responses to stress. Furthermore, the significant and potentially unique dysregulation of cellular redox systems in pathology, notably of the thioredoxin system, opens exciting vistas 
towards using TrxR-selective probes as tools both to study and ultimately to diagnose pathologies featuring redox dysregulation, such as autoimmune diseases and cancer. Finally, if such molecular probes are also modular designs, they offer to meet the full spectrum of needs in translational chemical biology: from redox-triggered diagnostics of diverse modalities (that are predictive of arbitrary cargo release), to the redox-triggered prodrugs that may allow targeting bioactivity selectively to the identified pathological tissues.

The selenenylsulfide-based probe RX1 exploits a "Selenium Required" mechanism-based design to perform as the first TrxR1-selective, modular, cargo-releasing cellular probe system. The cyclic topology of the stabilised selenenylsulfide substrates, and the mechanistic bias of the 5-exo-trig selenolate cyclisation, are both required for this performance. Its design is robust, yet its high selectivity for mammalian TrxR1 is accompanied by fast kinetics of processing and cargo release. Its true off-to-on optical performance (zero pre-activation background, to bright and photostable environment-independent fluorescence) and its cell-retained localisation permit cellresolved imaging with excellent signal-to-noise ratios. $\mathbf{R X 1}$ will thus be directly useful for redox biology studies quantifying TrxR1 activity in physiology and disease. Moreover, its high-quality performance in high-throughput screening can earn it a crucial place in development pipelines for selective cellular inhibitors of TrxR1: a goal of significant pharmaceutical interest, and one which we believe will more broadly enlighten the field towards the scope for high-selectivity inhibitors, whether tempered electrophiles or noncovalents.

The modularity of RX1 also suits it for flexible adaptation to TrxR1-triggered release of a range of cargos, far beyond the fluorogenic RX1 probe for cell culture. The use of a traceless solubiliser sidechain to ensure reliable and cargo-independent solubility is explicitly intended to favour just such adaptation. The design is suited for translation to any molecular cargo with unmaskable activity: of which the fluorogenic, indigogenic, or bioluminogenic probe possibilities in the section "Probe Design", and the accompanying drugreleasing prodrug possibilities, are just a small selection. Particularly given the strong pharmaceutical interest in TrxR-targeted and redoxactivated drugs in the inflammation, immunity, and cancer therapy fields, ${ }^{10}$ it will be exciting to test whether redox-responsive prodrug designs based around RX1-like selenenylsulfides can harness pathologically dysregulated TrxR1 activity to target drugs to diseased tissues. We also anticipate that improved RX1 analogues can be rationally designed to retain its specificity while installing enhanced reaction kinetics, alternative cargo delivery scope, and maximising its still-untested resistance to in vivo metabolism-based cargo release mechanisms, which will further promote its utility in biological and therapeutic applications. We thus expect that the RX1 system will impact both redox biology quantification/mapping studies, as well as translational research and therapy of locally-redox-dysregulated pathologies such as cancer, inflammation and auto-immune disorders.

The development and use of chemical probes for redox biology has been hampered by several systematic problems. ${ }^{72}$ Only very few redox-active trigger chemotypes have been explored; in our field, this lack of diversity is exacerbated by the overwhelming use of linear disulfides in probes (Fig. S1). The rational mechanism-based design of new redox-active motifs that are chemocompatible with the major biological redox systems, is urgently needed to drive innovation and research across the field. A second problem is the lack of clarity about what molecular information the known redox chemotypes do, or do not, provide. We believe that high-quality chemotypes should exhibit logical structure-activity relationships: for redox just as much as is accepted for ligand binding. A third problem is that redox selectivity testing is rarely as thorough as it could be. Selectivity is often stated despite it being obscure which cellular reductants have been controlled, and which have not; justifications of how much of a probe is claimed to be cellularly processed by the claimed species would be less ambiguous. The combination of these problems has resulted in a large number of redox probe reports claiming selectivity for various species, with even the same redox-active motifs being claimed as selective for a range of different redox species (Fig. S1), occasionally without or against supporting data or rationale. ${ }^{32}$

We also intended this probe research to test how such systematic problems may be overcome with chemistry. The two cyclic selenenylsulfide cores are new chemical species that open up broad possibilities for probe adaptations, which are easily accessible through their operationally simple, divergent, gram-scale syntheses. The exploration of the solubiliser sidechains, and the comparisons to related probe structures, confirms the rule of structure-activity relationships from cell-free to cellular applications. The battery of cell-free and cellular tests we have employed have stringently assayed all key aspects of redox probe performance, in particular to validate RX1's TrxR1selectivity. The comprehensive cell-free characterisations, using key redox-active species and proteins in their full interacting systems, tested several key features that cellular assays later confirmed by orthogonal measurements. RX1's cell-free resistance to physiological levels of monothiols over hours was indeed shown to depend on mechanism, since its thermodynamically identical but mechanistically differentiated G1 analogue is GSH-labile. The cell-free selenolthiol-selectivity of RX1 was shown by its nonresponse to the $\operatorname{TrxR}(\mathrm{U} 498 \mathrm{C})$ mutant, which until now was assumed to be similarly-reactive to most small molecule redox substrates including all that contain selenium ${ }^{32}$. The cellular counterpoint of these assays has been the validation of TrxR1 as the only relevant cellular target of RX1, by three independent biological and chemical methods (knockout/knock-in, chemical inhibition, and selenium starvation). Comparison to the performance of mixed-mode $\mathbf{G 1}$ (processed by both TrxR and thiols, as revealed by all three methods) further substantiated RX1's cellular selectivity, and provides a comprehensive and quantitative basis for reporting RX1 as a TrxR1-selective probe.

The use of chemical diversity, i.e. regioisomeric as well as disulfide and diselenide probes, as mechanistic, thermodynamic, and hydrolytic controls, has thus been a key element in testing and rationalising RX1's selenolthiol-selectivity. More broadly, the rationally-designed panel of selenium-containing motifs we present here complements the all-sulfur series we have reported ${ }^{17,23}$ to allow functional comparison and selectivity screening across a total of $>40$ biocompatible dichalcogenide redox probes, with a level of intercomparability and rigour that to our knowledge has not yet been attempted. It was our aim to increase the breadth and depth of this body of data, and particularly also to provide explicit mechanistic descriptions (Fig. S3S4) of how these small molecule probes have been designed for selectivity despite lacking the protein recognition features upon which genetic approaches can rely. Our hope is that by doing so, this research will both inspire new chemical approaches in the still-untapped field of redox chemical biology, and will also open doors for a range of powerful biological studies: revealing the key cellular redox networks in action, and addressing them through therapeutics targeted by the physiology and the pathology of TrxR1. 


\section{ONLINE CONTENT}

\section{Supporting Information}

The Supporting Information is available free of charge on the website. Synthesis, analysis, biochemical and biological evaluations (PDF)

\section{REFERENCES}

1. Arnér, E. S. J. \& Holmgren, A. Physiological functions of thioredoxin and thioredoxin reductase: Thioredoxin and thioredoxin reductase. European Journal of Biochemistry 267, 6102-6109 (2000).

2. Miller, C. G., Holmgren, A., Arnér, E. S. J. \& Schmidt, E. E. $\mathrm{NADPH}$-dependent and -independent disulfide reductase systems. Free Radical Biology and Medicine 127, 248-261 (2018).

3. Herrmann, J. M. \& Dick, T. P. Redox Biology on the rise. Biological Chemistry 393, 999-1004 (2012).

4. Jones, D. P. \& Sies, H. The Redox Code. Antioxidants \& Redox Signaling 23, 734-746 (2015).

5. $\quad$ Fridovich, I. et al. Antioxidant Enzymes. in Redox Biochemistry 49-134 (Wiley, 2007). doi:10.1002/9780470177334.ch3.

6. Meyer, A. J. \& Dick, T. P. Fluorescent Protein-Based Redox Probes. Antioxidants \& Redox Signaling 13, 621-650 (2010).

7. Lukyanov, K. A. \& Belousov, V. V. Genetically encoded fluorescent redox sensors. Biochimica et Biophysica Acta (BBA) - General Subjects 1840, 745-756 (2014).

8. Schwarzländer, M., Dick, T. P., Meyer, A. J. \& Morgan, B. Dissecting Redox Biology Using Fluorescent Protein Sensors. Antioxidants \& Redox Signaling 24, 680-712 (2016).

9. Yoo, M.-H. et al. Targeting Thioredoxin Reductase 1 Reduction in Cancer Cells Inhibits Self-Sufficient Growth and DNA Replication. PLOS ONE 2, e1112 (2007).

10. Labunskyy, V. M., Hatfield, D. L. \& Gladyshev, V. N. Selenoproteins: Molecular Pathways and Physiological Roles. Physiological Reviews 94, 739-777 (2014).

11. Cheng, Q., Sandalova, T., Lindqvist, Y. \& Arnér, E. S. J. Crystal structure and catalysis of the selenoprotein thioredoxin reductase 1.J Biol Chem 284, 3998-4008 (2009).

12. Gromer, S. et al. Active sites of thioredoxin reductases: Why selenoproteins? Proceedings of the National Academy of Sciences 100, 1261812623 (2003)

13. Arnér, E. S. J. Focus on mammalian thioredoxin reductases Important selenoproteins with versatile functions. $B B A$ 1790, 495-526 (2009).

14. Maroney, M. J. \& Hondal, R. J. Selenium versus sulfur: Reversibility of chemical reactions and resistance to permanent oxidation in proteins and nucleic acids. Free Radical Biology and Medicine 127, 228-237 (2018).

15. Reich, H. J. \& Hondal, R. J. Why Nature Chose Selenium. ACS Chem. Biol. 11, 821-841 (2016).

16. Sagemark, J. et al. Redox properties and evolution of human glutaredoxins. Proteins: Structure, Function, and Bioinformatics 68, 879-892 (2007).

17. Felber, J. G. et al. Selective, Modular Probes for Thioredoxins Enabled by Rational Tuning of a Unique Disulfide Structure Motif. J. Am. Chem. Soc. 143, 8791-8803 (2021).

18. Griffith, O. W. Biologic and pharmacologic regulation of mammalian glutathione synthesis. Free Radical Biology and Medicine 27, 922-935 (1999).

19. Hansen, R. E., Roth, D. \& Winther, J. R. Quantifying the global cellular thiol-disulfide status. Proceedings of the National Academy of Sciences 106, 422-427 (2009).

20. Pires, M. M. \& Chmielewski, J. Fluorescence Imaging of Cellular Glutathione Using a Latent Rhodamine. Org. Lett. 10, 837-840 (2008).

21. Lim, C. S. et al. Ratiometric Detection of Mitochondrial Thiols with a Two-Photon Fluorescent Probe. J. Am. Chem. Soc. 133, 11132-11135 (2011).
22. Butora, G. et al. Cyclic-Disulfide-Based Prodrugs for CytosolSpecific Drug Delivery. Angewandte Chemie International Edition 53, 14046 14050 (2014).

23. Felber, J. et al. Cyclic 5-membered disulfides are not selective substrates of thioredoxin reductase, but are opened nonspecifically by thiols. ChemRxiv (2020) doi:10.26434/chemrxiv.13483155.v1.

24. Burns, J. A. \& Whitesides, G. M. Predicting the stability of cyclic disulfides by molecular modeling: effective concentrations in thiol-disulfide interchange and the design of strongly reducing dithiols. J. Am. Chem. Soc. 112, 6296-6303 (1990).

25. Houk, J. \& Whitesides, G. M. Structure-reactivity relations for thiol-disulfide interchange. J. Am. Chem. Soc. 109, 6825-6836 (1987).

26. Lees, W. J. \& Whitesides, G. M. Equilibrium constants for thioldisulfide interchange reactions: a coherent, corrected set. J. Org. Chem. 58, 642-647 (1993).

27. Cherblanc, F. L., Chapman, K. L., Brown, R. \& Fuchter, M. J. Chaetocin is a nonspecific inhibitor of histone lysine methyltransferases. Nat. Chem. Biol. 9, 136-137 (2013).

28. Zong, L. et al. Epidithiodiketopiperazines: Strain-Promoted Thiol-Mediated Cellular Uptake at the Highest Tension. ACS Cent. Sci. 3, 449-453 (2017).

29. Zhang, L. et al. Highly Selective Off-On Fluorescent Probe for Imaging Thioredoxin Reductase in Living Cells. J. Am. Chem. Soc. 136, $226-$ 233 (2014).

30. Ma, H., Zhang, J., Zhang, Z., Liu, Y. \& Fang, J. A fast response and red emission probe for mammalian thioredoxin reductase. Chem. Commun. 52, 12060-12063 (2016).

31. $\mathrm{Li}, \mathrm{X}$. et al. A fast and specific fluorescent probe for thioredoxin reductase that works via disulphide bond cleavage. Nat Commun 10, 2745 (2019).

32. Lothrop, A. P., Ruggles, E. L. \& Hondal, R. J. No Selenium Required: Reactions Catalyzed by Mammalian Thioredoxin Reductase That Are Independent of a Selenocysteine Residue. Biochemistry 48, 6213-6223 (2009).

33. Singh, R. \& Whitesides, G. M. Degenerate intermolecular thiolate-disulfide interchange involving cyclic five-membered disulfides is faster by $\sim 10^{\wedge} 3$ than that involving six- or seven-membered disulfides. J. Am. Chem. Soc. 112, 6304-6309 (1990).

34. Laurent, Q., Sakai, N. \& Matile, S. The Opening of 1,2-Dithiolanes and 1,2-Diselenolanes: Regioselectivity, Rearrangements, and Consequences for Poly(disulfide)s, Cellular Uptake and Pyruvate Dehydrogenase Complexes. Helvetica Chimica Acta 102, e1800209 (2019).

35. Abegg, D. et al. Strained Cyclic Disulfides Enable Cellular Uptake by Reacting with the Transferrin Receptor. J. Am. Chem. Soc. 139, 231238 (2017)

36. Li, X. et al. A fast and specific fluorescent probe for thioredoxin reductase that works via disulphide bond cleavage. Nature Communications 10, 2745 (2019).

37. Suarez, S. I., Ambrose, R, Kalk, M. A. \& Lukesh III, J. C. Selenosulfides Tethered to gem-Dimethyl Esters: A Robust and Highly Versatile Framework for H2S Probe Development. Chemistry - A European Journal 25, 15736-15740 (2019).

38. Mafireyi, T. J. et al. A Diselenide Turn-On Fluorescent Probe for the Detection of Thioredoxin Reductase. Angewandte Chemie International Edition 59, 15147-15151 (2020).

39. Steinmann, D., Nauser, T. \& Koppenol, W. H. Selenium and Sulfur in Exchange Reactions: A Comparative Study. J. Org. Chem. 75, 66966699 (2010)

40. Wessjohann, L. A., Schneider, A., Abbas, M. \& Brandt, W. Selenium in chemistry and biochemistry in comparison to sulfur. 388, 997-1006 (2007).

41. Bachrach, S. M., Walker, C. J., Lee, F. \& Royce, S. Effect of Ring Strain on Nucleophilic Substitution at Selenium: A Computational Study of Cyclic Diselenides and Selenenyl Sulfides. J. Org. Chem. 72, 5174-5182 (2007)

42. Arai, K. et al. Modeling Thioredoxin Reductase-Like Activity with Cyclic Selenenyl Sulfides: Participation of an $\mathrm{NH} \cdots$ Se Hydrogen Bond 
through Stabilization of the Mixed Se-S Intermediate. Chem. Eur. J. 25, 12751-12760 (2019).

43. Hamsath, A. \& Xian, M. Chemistry and Chemical Biology of Selenenyl Sulfides and Thioseleninic Acids. Antioxidants \& Redox Signaling 33, 1143-1157 (2020).

44. Thorn-Seshold, O., Vargas-Sanchez, M., McKeon, S. \& Hasserodt, J. A robust, high-sensitivity stealth probe for peptidases. Chem. Commun. 48, 6253-6255 (2012).

45. Mata, G., do Rosário, V. E., Iley, J., Constantino, L. \& Moreira, R A carbamate-based approach to primaquine prodrugs: Antimalarial activity, chemical stability and enzymatic activation. Bioorganic \& Medicinal Chemistry 20, 886-892 (2012).

46. Kiernan, J. A. Indigogenic substrates for detection and localization of enzymes. Biotech Histochem 82, 73-103 (2007).

47. Kaskova, Z. M., Tsarkova, A. S. \& Yampolsky, I. V. 1001 lights: luciferins, luciferases, their mechanisms of action and applications in chemical analysis, biology and medicine. Chem. Soc. Rev. 45, 6048-6077 (2016).

48. Sharma, A. et al. Hypoxia-targeted drug delivery. Chem. Soc. Rev. 48, 771-813 (2019).

49. Twum, E. A. et al. Initial development of a cytotoxic amino-secoCBI warhead for delivery by prodrug systems. Bioorganic \& Medicinal Chemistry 23, 3481-3489 (2015).

50. Huang, Z., Terpetschnig, E., You, W. \& Haugland, R. P. 2-(2'Phosphoryloxyphenyl)-4(3H)-quinazolinone derivatives as fluorogenic precipitating substrates of phosphatases. Analytical Biochemistry 207, 32-39 (1992).

51. Prost, M., Canaple, L., Samarut, J. \& Hasserodt, J. Tagging Live Cells that Express Specific Peptidase Activity with Solid-State Fluorescence. ChemBioChem 15, 1413-1417 (2014).

52. Lukesh, J. C., Palte, M. J. \& Raines, R. T. A potent, versatile disulfide-reducing agent from aspartic acid. J. Am. Chem. Soc. 134, 4057-4059 (2012)

53. Diemer, V. et al. A cysteine selenosulfide redox switch for protein chemical synthesis. Nature Communications 11, 2558 (2020).

54. Metanis, N., Keinan, E. \& Dawson, P. E. Traceless Ligation of Cysteine Peptides Using Selective Deselenization. Angewandte Chemie International Edition 49, 7049-7053 (2010).

55. Lu, J. et al. Penultimate selenocysteine residue replaced by cysteine in thioredoxin reductase from selenium-deficient rat liver. The FASEB Journal 23, 2394-2402 (2009).

56. Cheng, Q. \& Arnér, E. S. J. Overexpression of Recombinant Selenoproteins in E. coli. in Selenoproteins (ed. Chavatte, L.) vol. 1661 231-240 (Springer New York, 2018).

57. Cheng, Q. \& Arnér, E. S. J. Selenocysteine Insertion at a Predefined UAG Codon in a Release Factor 1 (RF1)-depleted Escherichia coli Host Strain Bypasses Species Barriers in Recombinant Selenoprotein Translation. Journal of Biological Chemistry 292, 5476-5487 (2017).

\section{Methods}

For full details of all methods, syntheses, and assays, see the Supporting Information.

Materials. Chemical reagents were purchased from abcr, Acros, Alfa Aesar, Carbolution, Sigma-Aldrich, TCI and used without purification unless noted otherwise. Tetrahydrofuran (THF), dichloromethane (DCM) and dimethylformamide (DMF) for synthesis were stored under argon atmosphere over molecular sieves.

Analytics for synthesis. High resolution mass spectrometry (HRMS) was conducted using a Thermo Finnigan LTQ FT Ultra FourierTransform with electron spray ionisation (ESI), or a Finnigan MAT 95 with electron ionisation (EI). Nuclear magnetic resonance (NMR) spectroscopy was performed using a Bruker Avance (600/150 MHz, with TCI cryoprobe) or a Bruker Avance III HD Biospin $(400 / 100 \mathrm{MHz}$, with BBFO cryoprobe), and analysed with MestreNova 12. ${ }^{1} \mathrm{H}$-NMR spectra chemical shifts $(\delta)$ in parts per
58. Cheng, Q. et al. The selenium-independent inherent pro-oxidant NADPH oxidase activity of mammalian thioredoxin reductase and its selenium-dependent direct peroxidase activities. J Biol Chem $\mathbf{2 8 5}, 21708-21723$ (2010)

59. Rackham, O. et al. Substrate and inhibitor specificities differ between human cytosolic and mitochondrial thioredoxin reductases: Implications for development of specific inhibitors. Free Radical Biology and Medicine 50, 689-699 (2011).

60. Espinosa, B. \& Arnér, E. S. J. Thioredoxin-related protein of 14 $\mathrm{kDa}$ as a modulator of redox signalling pathways: TRP14 in redox signalling. British Journal of Pharmacology 176, 544-553 (2019).

61. Johansson, C., Lillig, C. H. \& Holmgren, A. Human mitochondrial glutaredoxin reduces S-glutathionylated proteins with high affinity accepting electrons from either glutathione or thioredoxin reductase. J Biol Chem 279, 7537-7543 (2004).

62. Peng, X., Xu, J. \& Arnér, E. S. J. Thiophosphate and selenite conversely modulate cell death induced by glutathione depletion or cisplatin: effects related to activity and Sec contents of thioredoxin reductase. Biochem J 447, 167-174 (2012)

63. Stafford, W. C. et al. Irreversible inhibition of cytosolic thioredoxin reductase 1 as a mechanistic basis for anticancer therapy. Sci. Transl. Med. 10, eaaf7444 (2018).

64. Mandal, P. K. et al. System xc- and Thioredoxin Reductase 1 Cooperatively Rescue Glutathione Deficiency*. Journal of Biological Chemistry 285, 22244-22253 (2010).

65. Peng, $\mathrm{X}$. et al. Sec-containing TrxR1 is essential for self-sufficiency of cells by control of glucose-derived H2O2. Cell Death Dis 5, e1235 (2014)

66. Peng, X. et al. Thioredoxin reductase 1 suppresses adipocyte differentiation and insulin responsiveness. Sci Rep 6, 28080 (2016).

67. Abdalbari, F. H. \& Telleria, C. M. The gold complex auranofin new perspectives for cancer therapy. Discover Oncology 12, 42 (2021).

68. Prast-Nielsen, S. et al. Inhibition of thioredoxin reductase 1 by porphyrins and other small molecules identified by a high-throughput screening assay. Free Radical Biology and Medicine 50, 1114-1123 (2011).

69. Eaton, J. K. et al. Selective covalent targeting of GPX4 using masked nitrile-oxide electrophiles. Nature Chemical Biology 16, 497-506 (2020).

70. Compound Library Screening with LOPAC ${ }^{\otimes} 1280$ Small Molecules.

71. Plemper, R. K. \& Cox, R. M. Biology must develop herd immunity against bad-actor molecules. PLOS Pathogens 14, e1007038 (2018).

72. Forman, H. J. et al. Even free radicals should follow some rules: A Guide to free radical research terminology and methodology. Free Radical Biology and Medicine 78, 233-235 (2015).

million $(\mathrm{ppm})$ relative to tetramethylsilane $(\delta=0 \mathrm{ppm})$ are reported using the residual protic solvent $\left(\mathrm{CHCl}_{3}\right.$ in $\mathrm{CDCl}_{3}: \delta=7.26 \mathrm{ppm}$, DMSO-d $\mathrm{d}_{5}$ in DMSO- $\mathrm{d}_{6}: \delta=2.50 \mathrm{ppm}, \mathrm{CHD}_{2} \mathrm{OD}$ in $\mathrm{CD}_{3} \mathrm{OD}$ : $\delta=3.31 \mathrm{ppm})$ as an internal reference. For ${ }^{13} \mathrm{C}-\mathrm{NMR}$ spectra, chemical shifts in ppm relative to tetramethylsilane $(\delta=0 \mathrm{ppm})$ are reported using the central resonance of the solvent signal $\left(\mathrm{CDCl}_{3}: \delta=\right.$ 77.16 ppm, DMSO-d $\left.6: \delta=39.52 \mathrm{ppm}, \mathrm{CD}_{3} \mathrm{OD}: \delta=49.00 \mathrm{ppm}\right)$ as an internal reference. Analytical high pressure liquid chromatography (HPLC) was conducted using an Agilent 1100 system with a Hypersil Gold HPLC column and DAD detector, or an Agilent 1200 SL system with a Hypersil Gold HPLC column, DAD detector, and low-resolution mass detection using a LC/MSD IQ mass spectrometer from Agilent (ESI), with water:MeCN eluent gradients containing $0.1 \%$ formic acid. Reactions were monitored by thin layer chromatography (TLC) on TLC plates (Si 60 F254 on aluminium sheets) provided by Merck $\mathrm{GmbH}$ and visualised by UV irradiation and/or $\mathrm{KMnO}_{4}\left(3.0 \mathrm{~g} \mathrm{KMnO}_{4}, 20 \mathrm{~g} \mathrm{~K}_{2} \mathrm{CO}_{3}, 0.30 \mathrm{~g} \mathrm{KOH}, 0.30 \mathrm{~L} \mathrm{H}_{2} \mathrm{O}\right)$. 
General synthetic procedures. Unless stated otherwise, all reactions were performed without air- or moisture-sensitivity precautions, and were stirred with Teflon-coated magnetic stir bars. For work under inert gas (nitrogen) atmosphere, a Schlenk apparatus equipped with a liquid nitrogen trap and a high vacuum pump from Vacuubrand were used. For solvent evaporation a Heidolph Laborota 400 equipped with a vacuum pump was used. Flash column chromatography was conducted under positive nitrogen pressure over Merck Ceduran $^{\circledast}$ Si 60 silica gel as stationary phase. TLC control, extractions and column chromatography were conducted using distilled, technical grade solvents.

Selenocyanation of alkyl mesylates: To the mesylate, dissolved in THF $(0.1 \mathrm{M})$, were added $\mathrm{NaI}$ (3.0 equiv.), 18-crown-6 ( 1.1 equiv.) and $\mathrm{KSeCN}$ ( 2.2 equiv.). The suspension was heated to $50{ }^{\circ} \mathrm{C}$ for $16 \mathrm{~h}$, then diluted with brine and DCM. The aqueous layer was extracted with DCM, and the combined organic phases were dried over $\mathrm{MgSO}_{4}$, filtered and concentrated in vacuo. Flash column purification of the oily orange crude afforded alkyl selenocyanates as colourless or pale yellow solids.

Base-mediated formation of selenenylsulfides/diselenides: Methanolic KOH (0.2 M, 2.0 equiv.) was added dropwise to a solution of thioacetate-selenocyanate or bis(selenocyanate) (1.0 equiv.) in THF $(0.05 \mathrm{M})$. Within seconds, the reaction turned from colourless to yellow and TLC indicated full conversion. The reaction was quenched with $\mathrm{NaHCO}_{3}$ (sat. aq.), and the aqueous layer was extracted with DCM. The combined organic layers were dried over $\mathrm{MgSO}_{4}$, filtered and concentrated in vacuo to give the selenenylsulfide/diselenide as a deeply coloured solid, used without further purification.

Probe formation by carbamate coupling between dichalcogenide secondary amines and PQ-chloroformate: The amine hydrochloride was suspended in DCM $(50 \mathrm{mM}) . \mathrm{NEt}_{3}$ (3.0 equiv.) was added, and the solution was added dropwise into a suspension of chloroformate PQ-OC $(\mathrm{O}) \mathrm{Cl}$ in DCM $(0.25 \mathrm{M})$. The resulting reaction was stirred for $1 \mathrm{~h}$, concentrated in vacuo yielding a pale yellow crude oil, and typically, flash column chromatography provided the target probe carbamates in excellent purity without contamination by residual fluorescent PQ-OH. For the solubilised piperazinamide probes including RX1, additional purification by preparative HPLC was conducted.

Cell-free screening. In vitro assays determining the $\mathbf{P Q}$ probes' redox properties are based on reduction-induced fluorophore release, quantified using a BMG FluoStar Omega plate reader (ex 355bp20, em 520lp). Black 96-well plates with black bottom were charged with probe and subjected to chemo- and bioreductant challenge (assay medium 1\% DMSO in TE buffer). PQ release was monitored at set times $(1 \mathrm{~min}, 15 \mathrm{~min}, 30 \mathrm{~min}, 1 \mathrm{~h}, 2 \mathrm{~h}, 3 \mathrm{~h}, 4 \mathrm{~h}, 5 \mathrm{~h}, 6 \mathrm{~h}$, $15 \mathrm{~h}$ ), in between which the plates were held in a thermostatted oven at $37^{\circ} \mathrm{C}$ in $\mathrm{H}_{2} \mathrm{O}$-saturated atmosphere. Raw data were processed using Microsoft Excel and GraphPad Prism. Fluorescence values $\mathrm{F}(\mathrm{t})$ were typically normalised per timepoint to the TCEP control $\mathrm{F}^{\mathrm{TCEP}}(\mathrm{t})$ (as a reference for fast, quantitative probe reduction and activation, so as to eliminate the delay of cyclisation kinetics from analysis). Data plotting was conducted using GraphPad Prism. Human recombinant thioredoxin 1 ( $\operatorname{Trx} 1)$ (lyophilized), human recombinant glutaredoxin 1 (Grx 1) (lyophilized from $10 \mu \mathrm{L}$ TE-buffer, $\mathrm{pH}$ 7.5), human thioredoxin reductase (TrxR) $(1.5 \mathrm{mg} / \mathrm{mL}$ in $50 \%$ glycerol/TE-buffer, $\mathrm{pH} 7.5$ ) and baker's yeast glutathione reductase (GR) (100 $\mu \mathrm{M}$ in 50\% glycerol/TE-buffer, $\mathrm{pH} 7.5)$ were obtained from IMCO Corp., Stockholm (Sweden).

Cellular assays. HeLa (DSMZ; ACC 57) and A549 (DSMZ; ACC 107) cell lines were purchased from the DSMZ. TrxR knockout and reference mouse embryonic fibroblasts (MEF) were a kind gift from Marcus Conrad. All cell lines are tested regularly for mycoplasma contamination and only mycoplasma negative cells are used in assays. Cells were grown in Dulbecco's modified Eagle's medium (DMEM; PAN Biotech) at $37^{\circ} \mathrm{C}$ under $5 \% \mathrm{CO}_{2}$ atmosphere, supplemented with $10 \% \mathrm{FBS}$, penicillin $(100 \mathrm{U} / \mathrm{mL})$, streptomycin $(100 \mu \mathrm{g} / \mathrm{mL})$ and $\mathrm{Na}_{2} \mathrm{SeO}_{3}(100 \mathrm{nM})$ unless stated otherwise. Selenium supplementation / starvation changes were always followed by at least 7 days of culturing the cells in the new media before performing any experiments. For assays, cells were typically seeded in 96well plates (black, Fluotrack, high binding; Greiner) in $100 \mu \mathrm{L}$ medium, probes were added (1\% DMSO final) using a D300e digital dispenser (Tecan), and timecourse fluorescence measurements were conducted using a BMG FluoStar Omega platereader (ex 355 bp 20, em 520lp). Cells were kept at $37^{\circ} \mathrm{C}$ under $5 \% \mathrm{CO}_{2}$ atmosphere between measurements.

Cellular quantitative high-throughput screening. $5 \mu \mathrm{L}$ of A549 cell suspension diluted to 1250 cells/well were dispensed into 1536 well black/clear flat bottom TC-treated microplates (Corning) using a Multidrop ${ }^{\mathrm{TM}}$ Combi Reagent Dispenser. Following $4 \mathrm{~h}$ incubation at $37^{\circ} \mathrm{C}$ to allow cells to adhere, $25 \mathrm{~nL}$ of LOPAC1280 compounds or controls (in DMSO) were spotted into wells using an Echo 525 Acoustic liquid handler, and cells were incubated for a further $1 \mathrm{~h}$. Then, $1 \mu \mathrm{L}$ of diluted $\mathbf{R X 1}$ were dispensed into each well using a BioRAPTR 2 FRD Dispenser (Beckman); plates were imaged at $1 \mathrm{~h}$ time intervals using an Envision plate reader $\left(\lambda_{\mathrm{ex}}=340 / 25 \mathrm{~nm}, \lambda_{\mathrm{em}}=520 / 25 \mathrm{~nm}, 10\right.$ flashes, bottom read $)$ during $4 \mathrm{~h}$ further incubation (plates kept at $37^{\circ} \mathrm{C}$ between readings).

\section{Author Contributions}

L.Z. performed synthesis, chemical analysis, chemoreductant and enzymatic cell-free studies, informatics, coordinated data assembly and wrote the manuscript. J.G.F. performed synthesis, chemical analysis, chemoreductant and enzymatic cell-free studies. K.S. performed enzymatic specificity screenings and cellular studies. L.P. performed cellular studies. D.C. performed LOPAC screening. M.S.M. performed synthesis and analysis. Q.C. expressed and purified recombinant TrxR and Trx isoenzymes. M.S. performed informatics. M.H. supervised LOPAC screening. E.S.J.A. supervised enzymatic specificity screenings and cellular studies and helped in interpretations of results and writing of the manuscript. J.T.-S. performed cellular studies and supervised cell biology. O.T.-S. designed the concept and experiments, performed informatics, supervised all other experiments, coordinated data assembly and wrote the manuscript. Correspondence and requests for materials should be addressed to O.T.-S.

\section{Competing Interests}

L.Z., J.G.F., and O.T.-S. are inventors on patent application EP21167187.0 covering compound structures reported in this paper. The authors declare no competing financial interest.

\section{Acknowledgments}

This research was supported by funds from the German Research Foundation (DFG: SFB 1032 project B09 number 201269156, SFB TRR 152 project P24 number 239283807, SPP 1926 project number 426018126, and Emmy Noether grant 400324123), LMUExcellent (Junior Researcher Fund), and the Munich Centre for NanoScience initiative 
(CeNS) to O.T.-S.; from Karolinska Institutet, the Knut and Alice Wallenberg Foundations (KAW 2015.0063 and KAW 2019.0059), the Swedish Cancer Society (CAN 2018/333 and 190330 Pj), the Swedish Research Council (2017-01872), the Hungarian Thematic Excellence Programme (TKP2020-NKA-26), the Hungarian National Research, Development and Innovation Office (ED_18-1-2019-0025), The Hungarian National Laboratories Excellence program (under the National Tumor Biology Laboratory project, NLP-17) and the Hungarian Ministry of Human Capacities (ÁEEK/41872-16/2020) to E.S.J.A; and the Intramural Research Program of the National Institutes of Health (NIH) National Center for Advancing Translational Sciences (NCATS) to M.H..

L.Z. thanks the Fonds der chemischen Industrie for generous financial support through a Kekule fellowship. J.G.F. thanks the Studienstiftung des deutschen Volkes for support through a $\mathrm{PhD}$ scholarship. L.P. thanks the GRK 2338 for support through a PhD scholarship. J.T.-S. thanks the Joachim Herz Foundation for fellowship support. We thank Marcus Conrad (Helmholtz Centre, Munich, DE) for MEF and knockout MEF cell lines; Carina Schmitt (LMU) for synthetic assistance; and Thomas Gudermann (LMU) for support through the GRK 2338. We thank the attendees of the SPP 1710 conference Thiol-Based Redox Switches in 2019, and in particular Christopher Lillig (Greifswald, DE), for their supportive, collegial, and helpfully challenging discussions.

This paper is dedicated to Yasuteru Urano, whose rigorous and farreaching developments towards translational applications of chemical biology probes are inspiring to us and to the field. 